\title{
Brown dwarfs and very low mass stars in the Hyades cluster: a dynamically evolved mass function ${ }^{\star}$
}

\author{
J. Bouvier ${ }^{1}$, T. Kendall ${ }^{2}$, G. Meeus ${ }^{3}$, L. Testi ${ }^{4,5}$, E. Moraux ${ }^{1}$, J. R. Stauffer ${ }^{6}$, D. James ${ }^{7}$, J.-C. Cuillandre ${ }^{8}$, J. Irwin ${ }^{9}$, \\ M. J. McCaughrean ${ }^{10}$, I. Baraffe ${ }^{11}$, and E. Bertin ${ }^{12, \star \star}$
}

${ }^{1}$ Laboratoire d'Astrophysique de Grenoble, Observatoire de Grenoble, BP 53, 38041 Grenoble, France e-mail: jbouvier@obs.ujf-grenoble.fr

2 Centre for Astrophysics Research, University of Hertfordshire, College Lane, Hatfield AL10 9AB, UK

3 Astrophysikalisches Institut Potsdam, An der Sternwarte 16, 14482 Potsdam, Germany

${ }^{4}$ Osservatorio Astrofisico di Arcetri, INAF, Largo E. Fermi 5, 50125 Firenze, Italy

5 European Southern Observatory, Karl Schwarzschild Str. 2, 85748 Garching bei München, Germany

${ }^{6}$ Spitzer Science Center, California Institute of Technology, 1200 East California Boulevard, Pasadena, CA 91125, USA

7 Department of Physics \& Astronomy, Box 1807 Station B, Vanderbilt University, Nashville, TN 37235, USA

8 CFHT Corporation, 65-1238 Mamalahoa Hwy, Kamuela, Hawaii 96743, USA

9 Harvard-Smithsonian Center for Astrophysics, 60 Garden Street MS-16, Cambridge, MA 02138, USA

10 School of Physics, Stocker Road, Exeter, EX4 4QL, UK

11 CRAL, Ecole Normale Supérieure, 46 allée d'Italie, 69364 Lyon Cedex 07, France

12 Institut d'Astrophysique de Paris, 98bis bd Arago, 75014 Paris, France

Received 21 December 2007 / Accepted 7 January 2008

\section{ABSTRACT}

\begin{abstract}
Aims. We conducted a search for brown dwarfs (BDs) and very low mass (VLM) stars in the 625 Myr-old Hyades cluster in order to derive the cluster's mass function across the stellar-substellar boundary.

Methods. We performed a deep $(I=23, z=22.5)$ photometric survey over $16 \mathrm{deg}^{2}$ around the cluster center and followed up with $K$-band photometry to measure the proper motion of candidate members and with optical and near-IR spectroscopy of probable BD and VLM members.

Results. We report the discovery of the first 2 BDs in the Hyades cluster. The 2 objects have a spectral type early- $T$ and their optical and near-IR photometry as well as their proper motion are consistent with them being cluster members. According to models, their mass is 50 Jupiter masses at an age of $625 \mathrm{Myr}$. We also report the discovery of 3 new very low mass stellar members of the cluster and confirm the membership of 16 others. We combine these results with a list of previously known cluster members to build the presentday mass function (PDMF) of the Hyades cluster from 50 Jupiter masses to $3 M_{\odot}$. We find the Hyades PDMF to be strongly deficient in very low mass objects and BDs compared to the IMF of younger open clusters such as the Pleiades. We interpret this deficiency as the result of dynamical evolution over the past few $100 \mathrm{Myr}$, i.e., the preferential evaporation of low mass cluster members due to weak gravitational encounters.

Conclusions. We thus estimate that the Hyades cluster currently hosts about 10-15 BDs, while its initial substellar population may have amounted to up to $150-200$ members.
\end{abstract}

Key words. stars: luminosity function, mass function - Galaxy: open clusters and associations: individual: Hyades (Melotte 25) stars: low-mass, brown dwarfs

\section{Introduction}

The determination of the initial mass function (IMF), i.e., the mass frequency distribution of objects formed in molecular clouds, yields strong constraints to star formation theories

\footnotetext{
* Appendices are only available in electronic form at http://www. aanda.org

$\star \star$ Partly based on observations obtained at the Canada-France-Hawaii Telescope (CFHT) which is operated by the National Research Council of Canada, the Institut National des Science de l'Univers of the Centre National de la Recherche Scientifique of France, and the University of Hawaii. Partly based on observations made with the Italian Telescopio Nazionale Galileo (TNG) operated on the island of La Palma by the Fundación Galileo Galilei of the INAF (Istituto Nazionale di Astrofisica) at the Spanish Observatorio del Roque de los Muchachos of the Instituto de Astrofisica de Canarias. Partly based on observations made with ESO Telescopes at the La Silla Observatories.
}

(Padoan \& Nordlund 2002; Bate \& Bonnell 2005; Larson 2005; Jappsen et al. 2005). In the last 10 years, the IMF has been derived from the most massive stars down to the substellar domain in a variety of environments: star forming regions (e.g. Luhman et al. 2003), young open clusters (e.g. Bouvier et al. 1998) and in the field (e.g. Reid et al. 1999). As brown dwarfs (BDs) continuously cool down as they evolve (Chabrier et al. 2000), they are brighter when younger. Thus, the IMF could be derived down to a mass of a few Jupiter masses in star forming regions (Lucas et al. 2006; Caballero et al. 2007) and down to about 30 Jupiter masses in rich young open clusters (Moraux et al. 2003, 2007; Barrado et al. 2004; de Wit et al. 2006). While a large number of field BDs have also been discovered within a few tens of parsecs from the Sun, the derivation of the field IMF below $0.1 M_{\odot}$ still remains uncertain due to the lack of knowledge of the ages of substellar objects (Chabrier 2002; Allen et al. 2005; Cruz et al. 2007). 
In a previous series of paper, we derived the lower mass function of a number of young open clusters down to about $30 M_{\text {Jup }}$ (see Moraux et al. 2005; Bouvier et al. 2005, for short reviews) and discussed the implications of an apparently universal cluster mass function for star formation scenarios (Moraux et al. 2007). Here, we report an extended, deep optical survey of the Hyades cluster aimed at detecting substellar objects and deriving the cluster's mass function across the stellar-substellar boundary. The motivations for this survey are twofold. Firstly, all previous searches for substellar objects in the Hyades have failed to report any positive BD detections (Reid \& Hawley 1999; Gizis et al. 1999; Dobbie et al. 2002). The lowest mass members reported so far includes LH $0418+13$, with a spectral type M 8.5 and an estimated mass of $0.083 M_{\odot}$ (Reid \& Hawley 1999), the $\simeq 0.081 M_{\odot}$ companions of a couple of $0.1 M_{\odot}$ Hyades probable members, LP 415-20 and LP 475-855 (Siegler et al. 2003), and an unresolved very low mass companion (0.070-0.095 $M_{\odot}$ ) in the spectroscopic binary RHy 403 (Reid \& Mahoney 2000). Yet, the discovery of BDs at an age of $625 \mathrm{Myr}$ (Perryman et al. 1998) would provide a unique benchmark to calibrate the substellar evolution models. Secondly, the Hyades cluster is dynamically evolved (Adams et al. 2002). A significant fraction of its initial low mass population is therefore expected to have drifted away beyond the cluster boundaries (e.g. Reid 1993). Deriving the lower mass function of such an evolved cluster would provide a direct measurement of the rate at which low mass cluster members evaporate and populate the field. In turn, this measurement would allow us to test the validity of $N$-body simulations of the dynamical evolution of young clusters (e.g. Kroupa 1995; Portegies Zwart et al. 2001).

The Hyades cluster (Melotte 25, $\alpha_{2000}=04^{\mathrm{h}} 26^{\mathrm{m}} 54^{\mathrm{s}}, \delta_{2000}=$ $\left.+15^{\circ} 52^{\prime} ; l=180.05^{\circ}, b=-22.40^{\circ}\right)$ is the closest rich open cluster to the Sun. Perryman et al. (1998) derived its main structural and kinematical properties based on Hipparcos measurements: a distance of $46.3 \pm 0.27 \mathrm{pc}$, an age of $625 \pm 50 \mathrm{Myr}$, a metallicity $[\mathrm{Fe} / \mathrm{H}]$ of $0.14 \pm 0.05$, a present-day total mass of about $400 M_{\odot}$, a tidal radius of $10.3 \mathrm{pc}$, a core radius of $2.5-3.0 \mathrm{pc}$ and negligible extinction on the line of sight. The large proper

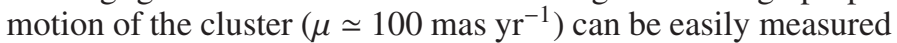
from imaging surveys over a timeframe of only a few years, which helps in assessing cluster's membership.

In Sect. 2, we describe the optical survey we performed over the central $16 \mathrm{deg}^{2}$ of the Hyades cluster, as well as follow-up $K$-band photometry and both optical and infrared spectroscopy. The proper motion of optically-selected candidate members is derived from optical and near-IR images obtained 2 to 3 years apart. In Sect. 3, we describe our selection of candidate members combining optical and infrared photometry, as well as proper motion measurements and follow up spectroscopy. We thus report 21 probable members, of which 5 are new and 2 are the first Hyades BDs, with an estimated mass of $\simeq 50 M_{\text {Jup }}$. In Sect. 4 , we discuss the spectral properties of the newly found low mass Hyades members, and derive a spectral type of $\mathrm{T} 1$ and $\mathrm{T} 2$ for the $2 \mathrm{BD}$ candidates. We proceed in deriving the present-day mass function of the Hyades cluster from 0.050 to $3.0 M_{\odot}$, which we find to be strongly deficient in very low mass stars and BDs compared to the mass function of the younger Pleiades cluster. We discuss this result in the light of $N$-body simulations of the dynamical evolution of young open clusters.

\section{Observations and data reduction}

We describe below the deep, wide field optical survey we performed on the Hyades cluster at CFHT in the $I$ and $z$ bands. We

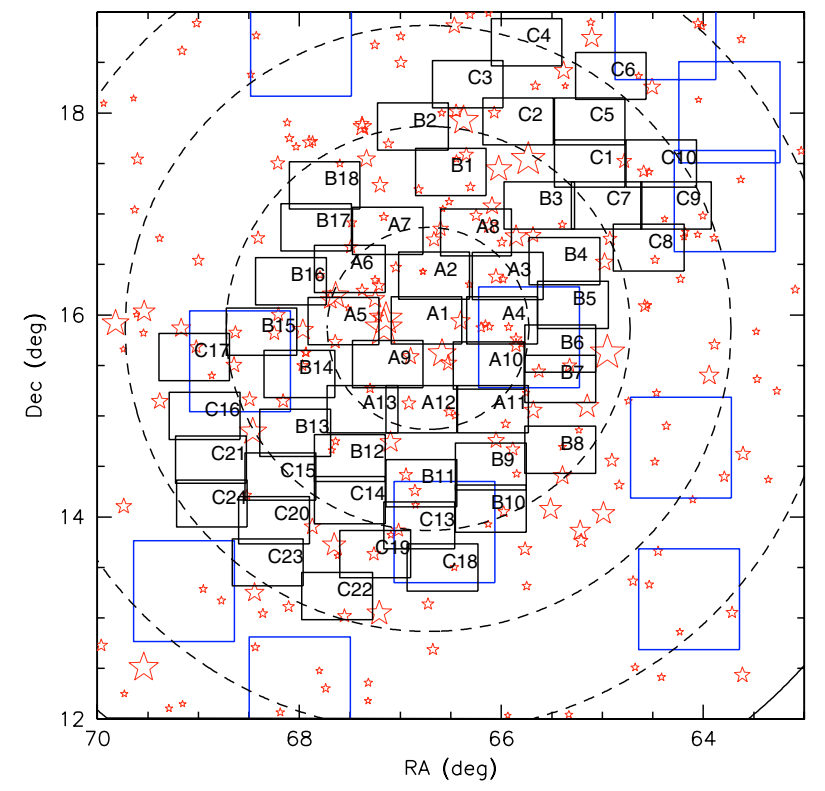

Fig. 1. The spatial distribution of CFHT12K Hyades fields is shown by rectangles labelled with their names (cf. Table A.1). Dashed concentric circles are spaced by $1^{\circ}$ around the cluster center. Star symbols (red) are Hyades probable members listed in Prosser \& Stauffer's database, with a size related to their luminosity. Large (blue) squares indicate the area previously surveyed by Dobbie et al. (2002).

also present follow up $K$-band imaging, as well as optical and infrared spectroscopy obtained for a subset of optically-selected cluster candidate members.

\subsection{The optical survey}

Wide-field optical images were obtained in the $I$ and $z$ bands with the CFHT $12 \mathrm{~K}$ camera, a mosaic of $12 \mathrm{CCD}$ arrays with a pixel size of $0.21^{\prime \prime}$ which provides a FOV of $42 \times 28^{\prime}$ (Cuillandre et al. 2000). The survey was performed during several service observing runs between September 2002 and January 2003, and consists of 53 mosaic fields covering a total of 17.3 square degrees over the cluster area. However, one CCD of the mosaic (CCD05) had serious cosmetics problems during these runs and yielded useless data, thus reducing the effective survey area by $1 / 12$. The dates of observation and the coordinates of the center of the mosaic fields are listed in Table A.1 in Appendix A. The area covered by the survey on the sky is illustrated in Fig. 1. The survey extends symmetrically around the cluster's center, along a 4 deg-wide stripe of constant galactic latitude, and up to 3 degrees away from the cluster center in galactic longitude.

Short and long exposures ( $10 \mathrm{~s}$ in $I$ and $z, 3 \times 300 \mathrm{~s}$ in $I$ and $3 \times 360 \mathrm{~s}$ in $z$ ) were obtained for each field. This resulted in a continuously sampled range of $I$ magnitudes from $I \sim 13$, the saturation limit of short exposures, to a detection limit of $I \sim$ 24. The survey is at least $90 \%$ complete down to $I \sim 23.0$ and $z \sim 22.5$, as computed from overlapping $12 \mathrm{~K}$ fields (see Moraux et al. 2003), a limit which varies only slightly with seeing conditions (0.6-0.8" FWHM, see Table A.1). Photometric standard fields (Landolt 1992) were observed several times per night, as part of the continuous monitoring of the CCD mosaic photometric zero points during service observing runs.

Individual images were processed through CFHT's Elixir reduction pipe-line (Magnier \& Cuillandre 2004) including bias subtraction, flat-fielding, fringe correction, scattered light 
correction in $z$-band, and astrometric calibration $\left(0.8^{\prime \prime} \mathrm{rms}\right)$. Multiple $I$ - and $z$-band dithered long exposures were then registered and co-added. PSF photometry was performed on both short and long exposures with a modified version of SExtractor (Bertin \& Arnouts 1996) from a PSF model computed with the PSFEx software (Bertin et al., in prep.). The resulting catalogues were photometrically calibrated using the $I$ - and $z$-band photometric zero points of the corresponding nights derived from the Landolt standard fields and extinction coefficients $k_{I}$ and $k_{z}$ of 0.04 and $0.03 \mathrm{mag} /$ airmass, respectively. In the $z$-band, the photometric zero points are computed from selected Landolt standards of spectral type A0. A few frames were taken through thin cirrus absorption (a few $0.01 \mathrm{mag}$ ). In this case, additional $I$ and $z$ exposures were obtained ( $30 \mathrm{~s}$ in $I, 36 \mathrm{~s}$ in $z$ ) for the same fields on subsequent photometric nights and used to recalibrate the original short and long exposure images.

Note that even though Landolt standards have been used to compute the photometric zero-points, the resulting $I$-band magnitudes are not in the Cousins system. This is due to the different passbands of the CFHT12K and Cousins filters which induces color terms, especially for very red objects. These color-terms can be estimated by integrating both filter passbands over Baraffe et al.'s (1998) model spectra. This yields: $I_{12} \mathrm{~K}=I_{\mathrm{c}}$ for $\left(I_{\mathrm{c}}-K\right) \leq 2.0$ and $I_{12 \mathrm{~K}}=I_{\mathrm{c}}-0.034 \times\left(I_{\mathrm{c}}-K\right)+0.068$ for $2.0 \leq\left(I_{\mathrm{c}}-K\right) \leq 6.0$. We have not applied these color terms on our photometry. Instead, $600 \mathrm{Myr}$ theoretical isochrones from the Lyon models were specifically computed for the $I$ - and $z$-band CFHT filters in order to provide a consistent comparison between models and observations in color-magnitude diagrams.

We found slight systematic differences between the photometric zero point of the various CCDs within the mosaic. The CCD-to-CCD zero point shifts were empirically estimated from the median $(I-z)$ color of all stellar-like objects in each CCD, with reference to CCD04. The distribution of color shifts, computed over all images, has a median value of -0.005 mag and a rms of 0.05 mag. The CCD-to-CCD zero point shifts were corrected for by applying them to the $z$-band magnitude of the objects in the output photometric catalogues. The I-band magnitude was left unchanged. Small overlaps between most of the observed fields eventually allowed us to check the quality of the photometric calibration. This was done by comparing the pair of magnitude measurements available for every star common to 2 overlapping fields. The rms accuracy of the $I$-band and $z$-band photometry is found to be of order of 0.06 mag for $I=18-20$ in long exposures, then increases to $0.08 \mathrm{mag}$ in the range $I=20-22$, and as the signal to noise ratio decreases further reaches $0.10 \mathrm{mag}$ at $I \sim 22.5$. Similar results, scaled to the corresponding magnitude range, are obtained for short exposure images.

The photometric catalogues originally contained nearly 2.3 million non saturated objects detected on the short and long exposures. Non stellar objects (galaxies, bad pixels, cosmics) were rejected from the photometric catalogues on the basis of the FHWM of their PSF. The seeing FWHM was measured on each image from plots of the object's magnitude against PSF $F W H M$. Stellar-like objects lie along a well defined vertical line in these plots, at the value of the seeing $F W H M$ (typically $0.6-0.8^{\prime \prime}$, i.e., $3-4$ pixels $F W H M$ ). Conservative limits typically between 50 and $150 \%$ of the seeing $F W H M$ were adopted to filter out galaxies (larger PSF FWHM) and bad pixels and cosmics (smaller PSF $F W H M$ ). Eventually, the output photometric catalogue contains over 930000 non saturated stellar-like objects detected in both $I$ - and $z$-bands over the 16 square degrees we surveyed down to a detection limit of $I \sim z \sim 24$. The

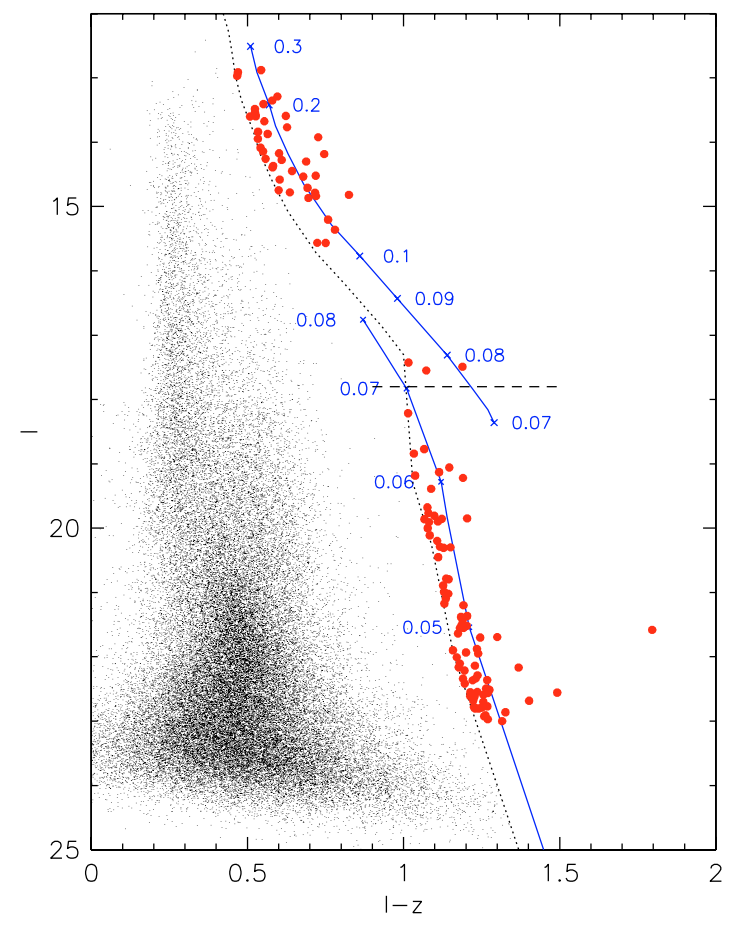

Fig. 2. $(I, I-z)$ color-magnitude diagram of stellar like objects detected on the Hyades CFHT12K frames. $600 \mathrm{Myr}$ NextGen (upper) and Dusty (lower) isochrones, shifted to the Hyades distance, are shown as solid lines and labelled with mass $\left(M_{\odot}\right)$. The horizontal dashed line illustrates the expected location of the stellar/substellar boundary along the cluster sequence. Hyades candidate members (large red dots) were selected to the right of the dotted line running close to the isochrones (see text). For clarity, only 1 in 10 objects of the background galactic population is shown leftwards of the selection line.

corresponding $(I, I-z)$ color magnitude diagram (CMD) is shown in Fig. 2. A total of 125 possible Hyades members were selected in this CMD from their location relative to model isochrones (see Sect. 3.1).

\subsection{Follow up K-band photometry}

Follow up $K^{\prime}$-band imaging was obtained for 108 of the 125 optically selected candidate members. About half of this sample was observed in Nov.04 and the other half in Nov. 05 using the $1 \mathrm{k} \times 1 \mathrm{k}$ CFHT IR camera (Starr et al. 2000). The exposure time varied from 0.5 to $14 \mathrm{~min}$ for candidates with I magnitudes ranging from 13 to 23. Each object was dithered on 7 positions on the detector, so that the median of the 7 images would provide an estimate of the sky background during the exposure. Individual images were dome flat fielded, sky subtracted, then registered and added to yield the final $K^{\prime}$-band image. $K$-band photometric standards from Hunt et al. (1998) were observed every couple of hours each night. Aperture photometry was performed on candidates and photometric standards. For 2 close visual binary systems (CFHT-Hy-15/17, CFHT-Hy-4/4c), a large aperture was first used to estimate the system's total flux, and PSF photometry was performed to derive the flux ratio of the system components.

The derived $K$-band photometric zero point is $22.86 \pm$ $0.03 \mathrm{mag}$, assuming an average extinction coefficient of $0.07 \mathrm{mag} /$ airmass. Slight photometric variations occurred during some nights due to varying extinction by thin cirrus. Images obtained on these nights were recalibrated using 2MASS images of the same fields. In this case, the photometric accuracy is of 


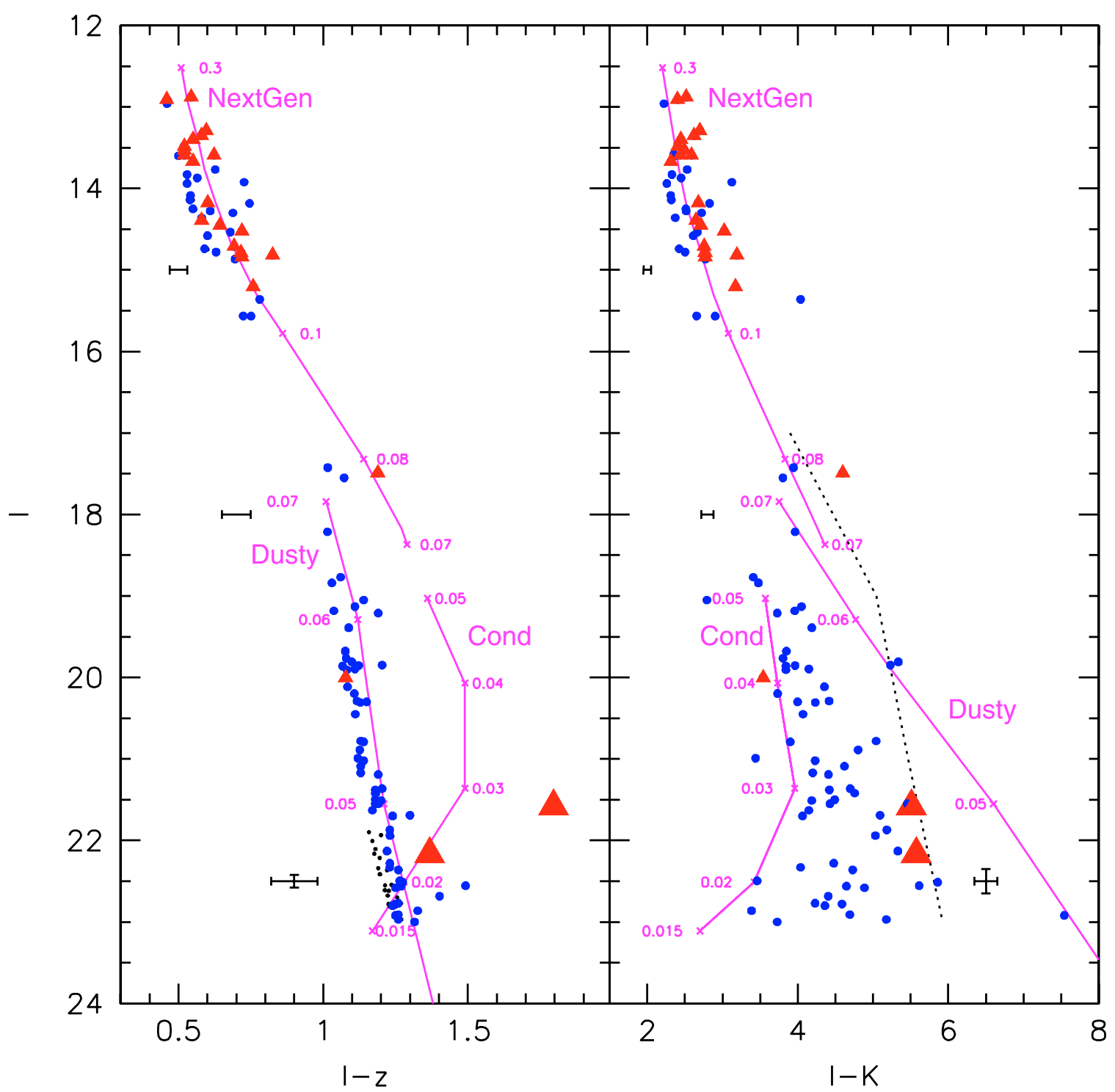

Fig. 3. Left: $(I, I-z)$ CMD of optically selected candidates. Right: $(I, I-K)$ CMD of optically selected candidated followed up with CFHT IR in the $K$-band. Small dots (left): 17 optically selected candidates without follow up IR photometry. Large dots: optically selected candidates whose proper motion is inconsistent with Hyades membership. Triangles: candidates whose proper motion is consistent with Hyades membership (see text). The 2 most promising substellar cluster candidates are shown by large triangles. NextGen $\left(0.07-0.3 M_{\odot}\right)$, Dusty $\left(0.04-0.07 M_{\odot}\right)$ and Cond $\left(0.015-0.05 M_{\odot}\right)$ isochrones are shown and labelled with mass. In the $(I, I-K)$ CMD, the dotted line indicates the locus of M8-T5 field dwarfs (from Dahn et al. 2002). The rms photometric error is shown as bars.

order of 0.05 mag. The other nights had photometric conditions and the derived $K$-band magnitudes have an accuracy of order of 0.02 mag. The $(I, I-z)$ and $(I, I-K)$ CMDs for the 108 candidates followed up in the $K$-band are shown in Fig. 3.

\subsection{Astrometry and proper motion}

The proper motion of optically selected Hyades candidates was computed from pairs of optical $(I, z)$ and infrared $(K)$ images obtained 2 or 3 years apart (Nov. 04, Nov. 05) under similar seeing conditions $\left(0.6-0.8^{\prime \prime} F W H M\right)$. The optical images were astrometrically calibrated through CFHT's Elixir pipe-line (Magnier \& Cuillandre 2004) with an accuracy of $0.8^{\prime \prime}$ rms. The infrared images were astrometrically calibrated using the WCStools package (Mink 2006) and 2MASS images as references. We found the CFHT IR detector to be aligned along the North-South direction to within $1 \mathrm{deg}$ and the pixel scale to be $0.211^{\prime \prime}$, as mentionned in CFHT IR's Reference Manual $\left(0.211 \pm 0.001^{\prime \prime}\right)$. Some CFHT IR images had not enough common objects with the shallower 2MASS images to allow a straight astrometric calibration through this method. In this case, we adopted the average orientation and plate scale derived for other CFHT IR images and simply calibrated the location of the center of the field.

Pairs of optical and infrared images where then matched by celestial coordinates to yield a list of common objects in the 2 images. The $(X, Y)$ location of the objects on both detectors was measured using IRAF/DAOFIND package. A geometrical transformation was then computed (IRAF/GEOTRANS) which maps the $(X, Y)$ coordinates of the optical detector into that of the IR detector. The geometrical transformation was derived from a set of typically 10 to 20 reference stars evenly distributed around 


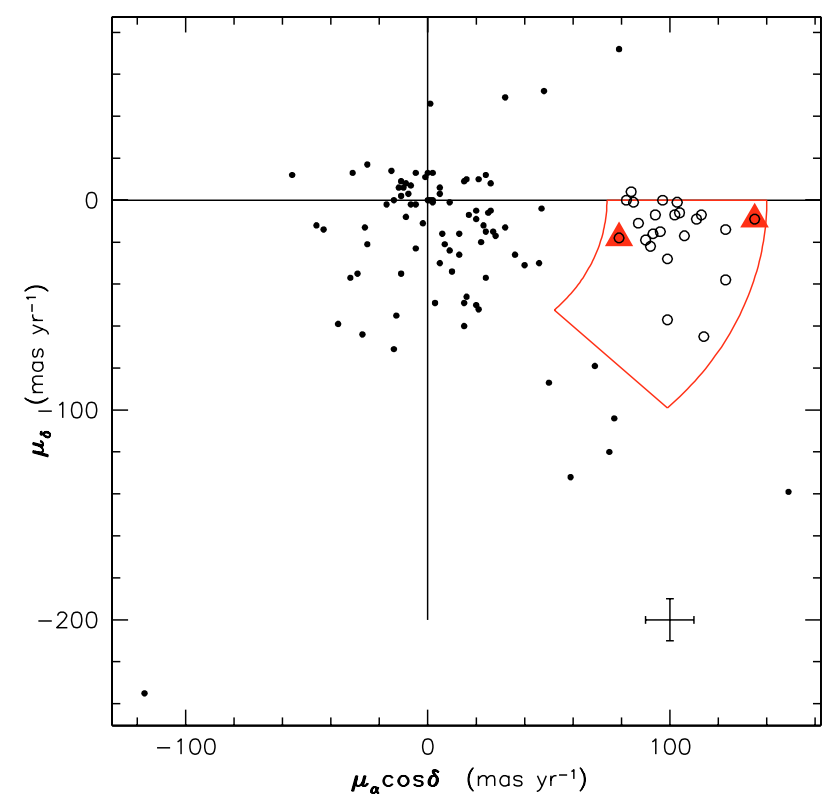

Fig. 4. Proper motion vector diagram for 107 optically selected candidates followed up in the $K$-band (see text). The expected proper motion for Hyades members is shown by the (red) box (Bryja et al. 2004). Within these boundaries, 23 optically selected candidates (empty circles) are found to share the proper motion of the cluster, including 2 BDs (large triangles). Typical rms errors on the ppm measurements are shown by a cross.

the Hyades candidate. The candidate itself is not included in the computation and an iterative procedure is applied to reject any reference star exhibiting high residuals. The accuracy of the geometrical transformation was typically 0.07 pixels rms.

The transformation was then applied to the Hyades candidate, mapping its optical $(X, Y)$ coordinates into the IR plane. The candidate proper motion was derived from the difference between the observed and mapped coordinates on the IR detector, divided by the time elapsed between the 2 images, and multiplied by the pixel scale. For each candidate, proper motion was measured using different sets of reference stars within the image, and from both the $I$ and $z$ images. The various measurements were always found to be consistent within errors and we finally considered their weighted mean as the best estimate of the candidate's proper motion.

The results are listed as $\mu_{\alpha \cos \delta}$ and $\mu_{\delta}$ in Tables 1 and D.1. The rms accuracy on the ppm measurement is usually of order of $10 \mathrm{mas} / \mathrm{yr}$. However, in some cases when the distribution of reference stars is asymetric relative to the candidate (i.e. when the optical candidate lies close to the CCD edge), higher systematic errors might be expected. Also, one candidate (HA13c08-18661) was too faint in $\mathrm{K}$ (a probable non member) to have its proper motion measured. The proper motion vector diagram of $107 \mathrm{op}-$ tically selected Hyades candidate members is shown in Fig. 4.

\subsection{Optical spectroscopy}

We obtain optical spectroscopy for 3 new Hyades low mass candidate members and for 2 other candidates recovered in our survey but previously classified as non members (see Sect. 3.1). Spectroscopic observations were performed from December 5 to 8, 2006 at ESO/NTT with EMMI spectrograph which provides echelle spectra covering the wavelength range from 580 to $990 \mathrm{~nm}$ at a resolution of 9800 . Using NOAO/IRAF, the spectral orders were extracted, flat-field corrected, wavelength calibrated using a Th-Ar lamp and corrected from the instrumental response computed from spectrophotometric standards observed during the same nights. Exposure times from 600 to $1200 \mathrm{~s}$ yielded a $\mathrm{S} / \mathrm{N}$ ratio of about 30 per resolution element at $850 \mathrm{~nm}$.

\subsection{Infrared spectroscopy}

The three faintest probable Hyades members (Table 1) were observed with the NICS near infrared spectrograph at the $3.58 \mathrm{~m}$ Telescopio Nazionale Galileo (TNG). We employed the high efficiency, very low resolution Amici spectroscopic mode, which yields a complete $0.85-2.45 \mu \mathrm{m}$ spectra with a resolving power of 50 (Baffa et al. 2001; Oliva et al. 2003). Observations were carried out in service mode by the TNG staff on Oct. 14 and 18, 2005. We used the $1^{\prime \prime}$ wide slit and total on-source integration times of 32, 56 and $40 \mathrm{~min}$ for CFHT-Hy-19, 20 and 21 respectively. The data reduction procedure closely followed the one described in Testi et al. (2001). Correction for telluric absorption was achieved using observations of the AS11 A0 star from the list of Hunt et al. (1998) obtained during the same nights at a similar airmass as our main targets and with an identical instrumental setup.

\section{Results}

We identified Hyades candidate members from their location in optical and IR color-magnitude diagrams (CMDs) and assess their membership status from proper motion measurements. We describe below the selection criteria we used and applied to all stellar like objects detected on the optical images.

\subsection{Probable Hyades members}

Possible Hyades members are selected from their location in the $(I, I-z)$ color magnitude diagram (CMD) relative to model isochrones. The 600 Myr NextGen (Baraffe et al. 1998) and Dusty (Chabrier et al. 2000) isochrones computed for CFHT $12 \mathrm{~K} I$ and $z$ filters were used. In order to account for photometric uncertainties and for the cluster's depth, the selection boundary was taken to lie 0.1 mag bluer than the isochrones, with the NextGen to Dusty transition occurring at $I \simeq 18.5$ mag. The $(I, I-z)$ CMD is shown in Fig. 2 together with the model isochrones labelled with mass shifted at a distance modulus of 3.33 for the Hyades cluster (Perryman et al. 1998). The selection resulted in 125 possible candidate members from $I \sim 13$ to 23 , covering the mass range from $0.3 M_{\odot}$ to about 45 Jupiter masses. The stellar/substellar boundary occurs at $I \sim 17.8$ in the CMD. Of the 125 possible candidates, 42 are low mass stars $\left(0.1-0.3 M_{\odot}\right), 4$ lie at the stellar/substellar boundary, and 79 lie in the substellar range $\left(M \leq 0.072 M_{\odot}\right)$. The list of optically selected candidates is given in Tables 1 and D.1.

Follow-up $K$-band imaging of 108 possible candidates provided additional membership criteria. The $(I, I-K) \mathrm{CMD}$ of these candidates is shown in Fig. 3. Most of the low mass stellar candidates qualify as possible members in this diagram as well, exhibiting $(I-K)$ colors consistent with model predictions. In contrast, a large fraction of the optically selected substellar candidates appear too blue compared to the Dusty model isochrones, which suggests they are older late type field dwarfs unrelated to the cluster. However, the model isochrones become increasingly uncertain at very low masses as dust develops and 
Table 1. Probable Hyades members based on photometry and proper motion.

\begin{tabular}{|c|c|c|c|c|c|c|c|c|c|}
\hline CFHT-Hy-\# & $\begin{array}{l}\text { Internal } \\
\text { name }\end{array}$ & RA(2000) & $\operatorname{Dec}(2000)$ & $I$ & $I-z$ & $I-K$ & $\begin{array}{l}\mu_{\alpha \cos \delta} \\
\text { (mas yr }\end{array}$ & $\begin{array}{c}\mu_{\delta} \\
-1)\end{array}$ & Other name ${ }^{a}$ \\
\hline CFHT-Hy-1 & HA10c00-33s & 42312.5 & 154247 & 12.87 & 0.54 & 2.5 & 111 & -9 & VA 262 \\
\hline CFHT-Hy-2 & $\mathrm{HC} 13 \mathrm{c} 03-43 \mathrm{~s}$ & 42724 & 1476 & 12.91 & 0.46 & 2.41 & 123 & -14 & VA 432 \\
\hline CFHT-Hy-3 & $\mathrm{HC} 06 \mathrm{c} 00-511 \mathrm{~s}$ & 41833.8 & 182153 & 13.29 & 0.59 & 2.69 & 114 & -65 & LP 414-158 \\
\hline CFHT-Hy-4 ${ }^{1}$ & $\mathrm{HC} 13 \mathrm{c} 11-268 \mathrm{~s}^{1}$ & 42822.4 & 134922 & 13.35 & 0.57 & 2.61 & 84 & 4 & HAN 430 \\
\hline CFHT-Hy-5 & HA2Ic06-310s & 42516.5 & 16187 & 13.4 & 0.55 & 2.45 & 87 & -11 & VA 352 \\
\hline СFHT-Hy-6 & HB10c09-178s & 42431.5 & 135541 & 13.48 & 0.52 & 2.41 & 97 & 0 & VA 326 \\
\hline СFHT-Hy-7 & HB8Ic01-117s & 42056.1 & 145133 & 13.53 & 0.52 & 2.5 & 106 & -17 & VA 203 \\
\hline CFHT-Hy-8 & HA11c00-112s & 4231.4 & 151341 & 13.59 & 0.52 & 2.43 & 103 & -1 & VA 260 \\
\hline CFHT-Hy-9 & HB12c04-432s & 43042.5 & 143941 & 13.59 & 0.62 & 2.59 & 113 & -7 & HAN 495 \\
\hline$\left(-^{2}\right.$ & HB6Ic04-77 $\mathrm{s}^{2}$ & 42232.8 & 155124 & 13.67 & 0.55 & 2.32 & 92 & -22 & VA 241) \\
\hline CFHT-Нy- $10^{3}$ & $\mathrm{HC} 04 \mathrm{c} 10-516 \mathrm{~s}^{3}$ & 42356.7 & 183819 & 14.17 & 0.6 & 2.68 & 102 & -7 & RHy 191 \\
\hline CFHT-Hy-11 & $\mathrm{HC} 16 \mathrm{c} 06-212 \mathrm{~s}$ & 43421 & 145116 & 14.39 & 0.57 & 2.65 & 82 & 0 & \\
\hline СFHT-Нy-12 & HB13c01-162s & 43116.3 & 15012 & 14.44 & 0.64 & 2.7 & 94 & -7 & \\
\hline CFHT-Hy-13 & $\mathrm{HC} 01 \mathrm{c} 00-279 \mathrm{~s}$ & 4195.7 & 173422 & 14.52 & 0.71 & 3.01 & 85 & -1 & \\
\hline СFHТ-Ну-14 & HA5Ic02-358s & 4304.2 & 1646 & 14.71 & 0.69 & 2.76 & 99 & -57 & RHy 281 \\
\hline СFHT-Ну- $15^{4}$ & HA2Ic03-783s ${ }^{4}$ & 4276.4 & 162548 & 14.78 & 0.71 & 2.76 & 93 & -16 & RHy 240 A \\
\hline СFHT-Нy-16 & $\mathrm{HC} 19 \mathrm{c} 03-841 \mathrm{~s}$ & 4292.9 & 133759 & 14.81 & 0.82 & 3.19 & 104 & -6 & LH 91, LHD 0426+1331 \\
\hline CFHT-Hy- $17^{4}$ & HA2Ic03-781 $\mathrm{s}^{4}$ & 4276.6 & 162546 & 14.83 & 0.71 & 2.76 & 96 & -15 & RHy 240B \\
\hline CFHT-Hy-18 & $\mathrm{HC} 09 \mathrm{c} 09-231 \mathrm{~s}$ & 41732.2 & 165659 & 15.2 & 0.75 & 3.17 & 123 & -38 & RHy 138 \\
\hline CFHT-Hy- $19^{5}$ & $\mathrm{HC} 08 \mathrm{c} 07-331 \mathrm{~s}^{5}$ & 41724.8 & 163436 & 17.49 & 1.18 & 4.59 & 99 & -28 & 2MASSW J0417247+163436 \\
\hline CFHT-Hy-20 6 & $\mathrm{HC} 22 \mathrm{c} 09-17351^{6}$ & 43038.7 & 13957 & 21.58 & 1.79 & 5.5 & 135 & -9 & \\
\hline CFHT-Hy-217 & HA9Ic04-13461 ${ }^{7}$ & 42922.7 & 153529 & 22.16 & 1.36 & 5.57 & 79 & -18 & \\
\hline
\end{tabular}

${ }^{a}$ Ref.: VA = van Altena (1969); LP = Luyten et al. (1981); HAN = Hanson (1975); RHy = Reid (1992); LH = Leggett \& Hawkins (1988); LHD = Leggett et al. (1994); 2MASS = Gizis et al. (1999).

${ }^{1}$ A faint companion is seen 3.2" away from this Hyades probable member but its $I-K$ color and proper motion indicates a background star.

${ }^{2}$ Classified as a non member by Reid (1993). Spectroscopically confirmed as a non-member in this study (see text).

${ }^{3}$ Classified as a non member by Reid (1993). Spectroscopically confirmed as a member in this study (see text).

${ }^{4}$ CFHT-Hy-15 and 17 form a visual pair (RHy $240 \mathrm{AB}$ ).

${ }^{5}$ Classified as a non member by Gizis et al. (1999), see text.

${ }^{6}$ This probable Hyades BD was also observed in the $J$ and $H$ bands, yielding $(J-H, H-K)=(0.51,0.43)$.

${ }^{7}$ This probable Hyades BD was also observed in the $J$ and $H$ bands, yielding $(J-H, H-K)=(1.12,0.77)$.

may condense in the lower atmospheric layers, which results in a sudden change of the $I-K$ index towards much bluer colors. The location of low mass substellar cluster members in the $(I, I-K)$ diagram may thus span a large range of colors, from the Dusty isochrone bluewards. Chabrier et al. (2000) predict that the transition between the Dusty and Cond models should occur at a temperature of $1300-1400 \mathrm{~K}$, which corresponds to a mass of 40 Jupiter masses for Hyades members.

As an additional membership criterion, proper motion was computed for all the candidates followed up in the $K$-band, except one (HA13c08-18661) which was too faint in K and therefore a probable non member. The proper motion vector diagram of 107 optically selected candidates is shown in Fig. 4. While most of the candidates scatter near the origin, with relatively small proper motion and thus most likely background field contaminants, 23 candidates have a proper motion consistent with Hyades membership. The ppm vector diagram also reveals 7 high proper motion objects $(\mu>100$ mas/yr), most likely foreground late-type field dwarfs.

In order to analyse the distribution of objects in the ppm diagram, we built a synthetic ppm vector diagram from simulated observations spanning $1 \mathrm{sq}$ deg in the direction of the Hyades cluster. The simulated sample was obtained from the Besancon model of galactic structure and kinematics (Robin et al. 2003). We extracted from this sample all the objects lying in the same part of the $(I, I-K)$ CMD as the optically selected Hyades candidates. The ppm vector diagram of the resulting synthetic subsample strongly ressembles the observed one, with most objects scattered around the origin with a slightly elongated distribution towards the south-east direction, and a few high velocity foreground dwarfs. No object from the simulated sample is found to lie within the boundary of the Hyades cluster proper motion. This indicates that the contamination of the observed ppm vector diagram by foreground field dwarfs at the expected location of Hyades members is small. We find only one optically selected candidate (HA12c07-1011) with a proper motion consistent with Hyades membership which is too blue in the $(I, I-K)$ CMD to be a member (cf. Table D.1). We classified it as a non member.

Eventually, 22 candidates consistently qualify as probable Hyades members on the basis of their optical photometry, their $(I-K)$ color and their proper motion. Their properties are listed in Table 1 and their spatial distribution is shown in Fig. 5. Of these, 14 were already listed as probable Hyades members in Prosser \& Stauffer's Open Cluster Database ${ }^{1}$ (P\&S database) based on various criteria and are confirmed here. Their alternate names are given in Table 1. Another 3 probable members in our survey had been previously reported but classified as probable non members. One (CFHT-Hy-10 = RHy 191) is listed in the P\&S database and classified as a probable non member based on the lack of $\mathrm{H}_{\alpha}$ emission in a low resolution spectrum (Stauffer's priv. comm., quoted in Reid 1993). The higher resolution

\footnotetext{
1 Open Cluster Database, as provided by C.F. Prosser and J.R. Stauffer, and which currently may be accessed at http://www.noao.edu/ noao/staff/cprosser/, or by anonymous ftp to 140.252.1.11, cd /pub/prosser/clusters/
} 


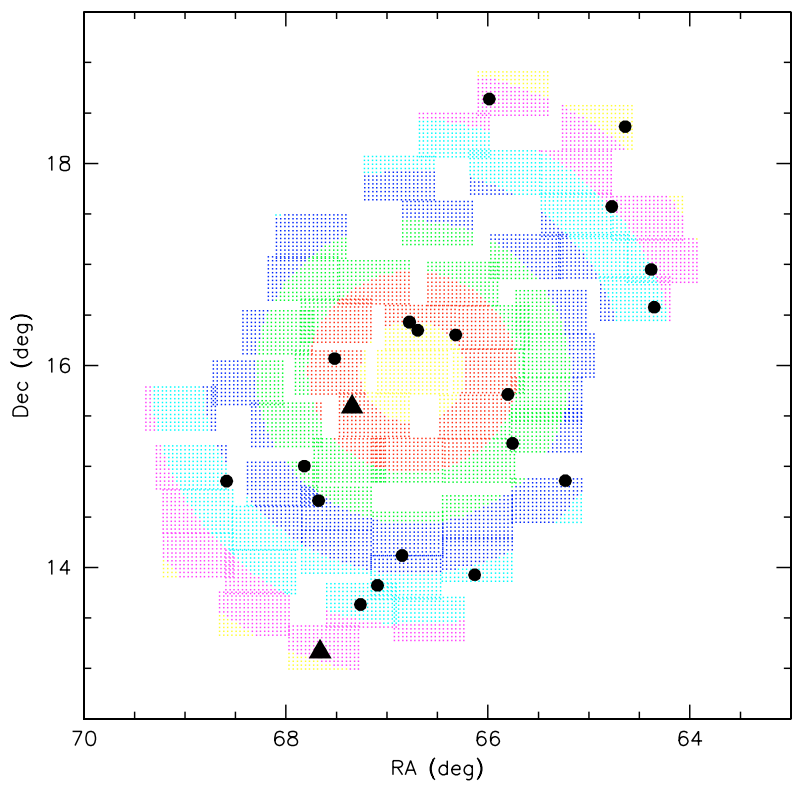

Fig. 5. The spatial distribution of probable Hyades members reported from our survey. Low-mass stellar members are shown as large dots, and the 2 BDs by triangles. The separation of the 2 components of the visual binary CFHT-Hy-15/17 (RHy 240AB) has been exagerated for clarity. The background shows the location of the $12 \mathrm{~K}$ fields (the color code illustrates $0.5 \mathrm{deg}$-wide rings around the cluster center).

spectrum we report for this object exhibits weak $\mathrm{H}_{\alpha}$ emission, with $E W\left(\mathrm{H}_{\alpha}\right)=3 \AA$, and deep NaI 8183, $8195 \AA$ lines, consistent with membership (cf. Fig. 6). Another candidate (HB6Ic04_77s = VA 241), also listed in the P\&S database, was classified as a non member by Reid (1993) based on " $(V-I)$ colours inconsistent with Hyades membership". The high resolution spectrum we obtain for VA 241 shows $\mathrm{H}_{\alpha}$ in absorption and shallow NaI doublet lines, a non member indeed (Fig. 6). The last one (CFHT-Hy-19) is located close to the stellar/substellar boundary and has previously been reported by Gizis et al. (1999) from the 2MASS survey of the Hyades cluster. This candidate is discussed in more details in the next section, as the evidence for membership is somewhat inconclusive. The remaining 5 probable members we report are new. They include 3 low mass stars $\left(\sim 0.14 M_{\odot}\right)$ and 2 objects well within the substellar regime $\left(\sim 0.050 M_{\odot}\right)$. The spectra of the 3 new low mass stars exhibit $\mathrm{H}_{\alpha}$ emission with equivalent widths between 4 and $6 \AA$, and deep NaI doublet, fully consistent with membership (cf. Fig. 6).

In addition, we found during this survey an extremely red object, HB6Ic09_3221, with $I=22.92$ and $I-K=7.55$ ! The object appears point like in the IzK images but is not a Hyades member according to its proper motion (Table D.1). We note that this object lies only $3^{\prime}$ away from the Class 0 protostar IRAM 04191+1522 (André et al. 1999) and from the Class I protostar IRAS 04191+1523 (Lee et al. 2002), both associated to the molecular condensations MC 18a and 18b (Onishi et al. 2002). This indicates that star formation is still active in this area located at the outskirt of the Taurus molecular cloud. HB6Ic09_3221, whose proper motion is consistent with Taurus membership (Frink et al. 1997), could thus be a new, selfembedded Class I source. However, there are no 2MASS nor IRAS counterparts at this location, nor is it detected by Spitzer at 24 and $70 \mu \mathrm{m}$ (Ph. André, priv. comm.).
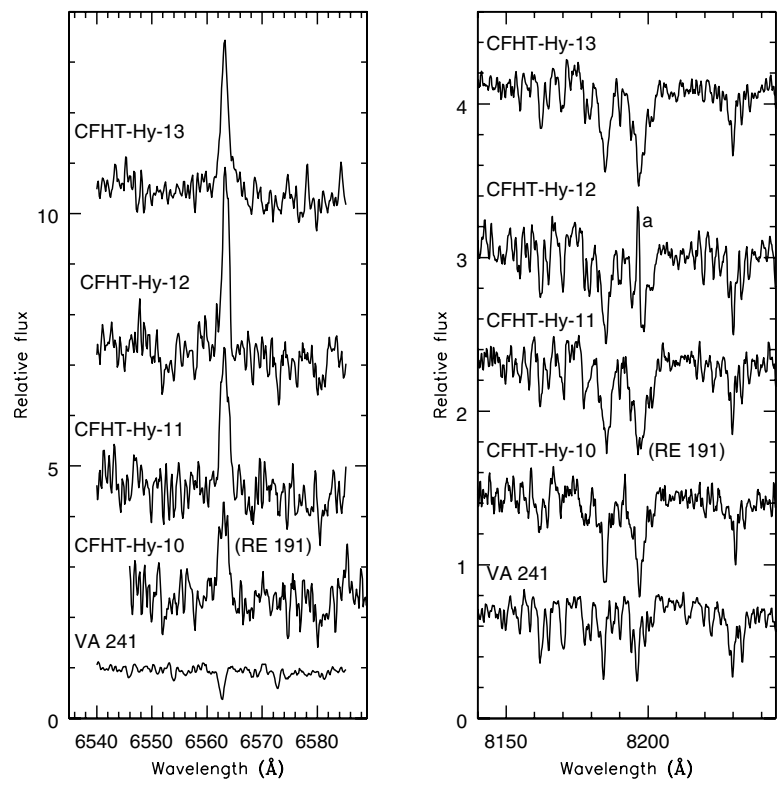

Fig. 6. Optical spectra of 5 optically-selected Hyades very low mass stellar candidate members. The $\mathrm{H}_{\alpha}$ region is shown in the left panel, the NaI doublet in the right panel. The NaI doublet $8195 \AA$ line of CFHT-Hy-12 is affected by a bad pixel, labelled with "a".

\subsection{Comparison with previous Hyades surveys}

In order to check the consistency of our membership classification of the 125 optically selected candidates, we searched the literature to compare our results with those of previous deep surveys of the Hyades cluster.

Dobbie et al. (2002) conducted a wide (10.5 sq deg) optical $(I, z)$ survey of the Hyades cluster down to a completeness limit of $I \sim 20.3 \mathrm{mag}$. They report 20 possible candidate members, of which they confirm only one as being a (previously known) proper motion low mass member (h5078e = RHy 297). Of the 20 candidates they report, 8 fall in the area we surveyed. Of these, 4 are too blue in the $(I, I-z)$ CMD to be selected as possible members (h8644b, h1272b, h8711b, h5044b), and 2 are saturated on our images (h9448b, h4239b). The 2 remaining ones are recovered here as possible members in the $(I, I-z)$ CMD but are eventually rejected on the basis of their discrepant proper motion (HB7Ic04_145s = h4046b; HB7Ic11_463s = h7334b).

Reid \& Hawley (1999, RH99) reported low resolution follow up spectroscopy of 12 possible low mass candidate members identified by Leggett \& Hawkins (1989, LH) from a Schmidt survey of the central regions of the cluster. Based on radial velocity measurements, they find that only one of the 12 stars is a probable low mass cluster member (LH 0418+13). Of the $12 \mathrm{LH}$ objects, 6 fall in the cluster area we surveyed. Of these, 4 are too blue in our $(I, I-z)$ CMD to be selected as possible cluster members $(\mathrm{LH} 0427+12,0418+15,0419+15,0420+15)$. Another one (LH $0422+17$ = HB1Ic01_3861) was selected as a possible member from its location in the $(I, I-z)$ CMD but its proper motion proved inconsistent with membership. RH99 also measured a discrepant radial velocity for this object, which appears to be an unrelated field dwarf. The last object (LH 0424+15) falls on a bad column of a CCD preventing us from deriving its photometry.

Gizis et al. (1999, GRM99) searched for very low mass stars and brown dwarfs in the central region of the Hyades cluster using the 2MASS survey. They selected 40 possible candidates, including 29 new ones, from their infrared colors corresponding 
to spectral types M8-L4. From the follow up low resolution spectroscopy they performed, none of these candidates appear to be cluster members. Eleven GRM99 new candidates fall in the area covered by our survey. Of these 11, 10 are too blue in the $(I, I-z)$ CMD to be selected as possible cluster members (2MASSW J0434462+144802, J0435489+153719, $\mathrm{J} 0431500+152814 ， \mathrm{~J} 0431322+152620 ， \mathrm{~J} 0431420+162232$, $\mathrm{J} 0421175+153003 ， \mathrm{~J} 0421521+151941 ， \mathrm{~J} 0424045+143129$, J0430232+151436, J0423242+155954). The last one, however, CFHT-Hy-19 = 2MASSW J0417247+163436, lies close to the stellar/substellar boundary in Fig. 2. We classify it as a probable cluster member based on its optical photometry, $(I-K)$ color and proper motion, all being consistent with membership. Gizis et al. (1999) dismissed this candidate as a cluster member based on its low resolution spectrum (Sp.T. M8) exhibiting weak NaI $8200 \AA$ equivalent width, suggestive of low surface gravity. They also fail to detect $\mathrm{H}_{\alpha}$ in emission in their low resolution spectrum. Comparing with theoretical models, they estimate a surface gravity of $\log g=4.4-4.7$, somewhat lower than expected for Hyades members $(\log g \sim$ 5.0). They conclude it is presumably a young (10-30 Myr), background $(d \sim 150-200 \mathrm{pc})$ substellar object $\left(M \leq 0.04 M_{\odot}\right)$, possibly associated with the nearby Taurus star forming region. We find this possibility unlikely however, as the proper motion we measure for this candidate ([99, -28] mas/yr) is much larger than the proper motion of Taurus SFR members (typically, [10, -20] mas/yr, Frink et al. 1997). Furthermore, the candidate's proper motion is fully consistent with Hyades membership. Hence, pending additional evidence for (or against) membership, we retain this candidate as a probable member.

Finally, Prosser \& Stauffer's Open Cluster Database lists 147 probable members with $V \geq 15.3$ ( $I \geq 12.5)$. Of these, 30 lie in the area covered by our survey: 14 are recovered and confirmed here as members (see Table 1, VA 262, VA 432, LP 414-158, HAN 430, VA 352, VA 203, VA 326, VA 260, HAN 495, RHy 281, RHy 138, RHy 240A, RHy 240B, LH 91); 9 others are saturated on our short exposure images, with $I$ magnitudes in the range from 12.52 to 13.36 and are therefore not considered in our study (VA 329, VA 213, VA 216, VA 94, VA 362, LP 415-875, VA 763, VA 127, VA 368, all of which except VA 329 have been spectroscopically confirmed as members, e.g., Reid \& Mahoney 2000); another one is HAN 150 (VA 95, RHy 133, HC08c00_261s) which we do recover in our survey but has too blue an $(I-z)$ color to be a candidate member $(I=$ $15.72, I-z=0.4)$. It was already rejected by Reid et al. (1993) based on its too blue $(V-I)$ color. The 6 remaining objects are the RH99 candidates discussed above, none of which qualify as a member in our study. Hence, we recover all the candidate probable members listed in the P\&S database which lie in the area covered by our survey and provide a new membership assessment for most of them. Another 6 objects listed as probable members in the P\&S database lie in the spatial limit of our survey but fall in gaps between our fields, as we avoided the immediate vicinity of bright stars. These are RHy 159, RHy 228, RHy 230, RHy 287 (Reid 1992) and LH 0416+16, LH 0429+15 (Legget \& Hawkins 1988). Two of these (RHy 228 and RHy 230) have been confirmed as members (Reid et al. 1995; Reid \& Mahoney 2000), 2 others (RHy 159, RHy 287) have not been followed up (Reid 1992), and the 2 remaining ones have been shown to be non-members (Reid \& Hawley 1999). Conversely, as discussed above (see Sect. 3.1), we recovered 2 objects, previously classified as non-members in the P\&S database, one which we confirm as being a non-member (VA 241), the other which we classify as a bona fide Hyades member (CFHT-Hy-10 = RHy 191).
Table 2. Mass estimates for the $12 \mathrm{~K}$ Hyades members.

\begin{tabular}{lllllll}
\hline \hline $\begin{array}{l}\text { CFHT- } \\
\text { Hy-\# }\end{array}$ & $I$ & $\begin{array}{l}\text { Mass }(I) \\
\left(M_{\odot}\right)\end{array}$ & $z$ & $\begin{array}{l}\operatorname{Mass}(z) \\
\left(M_{\odot}\right)\end{array}$ & $K$ & $\begin{array}{l}\operatorname{Mass}(K) \\
\left(M_{\odot}\right)\end{array}$ \\
\hline 1 & 12.87 & 0.256 & 12.33 & 0.258 & 10.37 & 0.292 \\
2 & 12.91 & 0.251 & 12.45 & 0.244 & 10.5 & 0.273 \\
3 & 13.29 & 0.214 & 12.7 & 0.217 & 10.6 & 0.258 \\
4 & 13.35 & 0.208 & 12.78 & 0.209 & 10.74 & 0.239 \\
5 & 13.4 & 0.203 & 12.85 & 0.201 & 10.95 & 0.214 \\
6 & 13.48 & 0.196 & 12.96 & 0.192 & 11.07 & 0.2 \\
7 & 13.53 & 0.192 & 13.01 & 0.188 & 11.03 & 0.205 \\
8 & 13.59 & 0.188 & 13.07 & 0.183 & 11.16 & 0.192 \\
9 & 13.59 & 0.188 & 12.97 & 0.191 & 11 & 0.208 \\
10 & 14.17 & 0.151 & 13.57 & 0.150 & 11.49 & 0.164 \\
11 & 14.39 & 0.141 & 13.82 & 0.138 & 11.74 & 0.146 \\
12 & 14.44 & 0.139 & 13.8 & 0.139 & 11.74 & 0.146 \\
13 & 14.52 & 0.136 & 13.81 & 0.138 & 11.51 & 0.162 \\
14 & 14.71 & 0.128 & 14.02 & 0.129 & 11.95 & 0.133 \\
15 & 14.78 & 0.126 & 14.07 & 0.127 & 12.02 & 0.129 \\
16 & 14.81 & 0.125 & 13.99 & 0.130 & 11.62 & 0.154 \\
17 & 14.83 & 0.125 & 14.12 & 0.125 & 12.07 & 0.126 \\
18 & 15.2 & 0.113 & 14.45 & 0.113 & 12.03 & 0.128 \\
19 & 17.49 & 0.074 & 16.31 & 0.077 & 12.9 & 0.094 \\
20 & 21.58 & 0.050 & 19.79 & 0.052 & 16.08 & 0.039 \\
21 & 22.16 & 0.048 & 20.8 & 0.049 & 16.59 & 0.035 \\
\hline
\end{tabular}

\section{Discussion}

\section{1. $L$ and $T$ dwarfs in the Hyades cluster}

We report here the first 2 Hyades BD candidates (CFHT-Hy$20,21)$ as well as a previously detected very low mass object (CFHT-Hy-19) close to the stellar-substellar boundary. The 3 objects are strong candidate cluster members as their optical/infrared luminosity and colors are consistent with membership, as is their proper motion. The 2 BDs are well within the substellar domain with an estimated mass of about 50 Jupiter masses (see Table 2) while the lowest mass star has an estimated mass around $0.08 M_{\odot}$. A finding chart for the 2 substellar objects is provided in Fig. B.1. According to models, at an age of $625 \mathrm{Myr}$, these objects should have an effective temperature around $1600 \mathrm{~K}$ for the $2 \mathrm{BDs}$ and around $2400 \mathrm{~K}$ for the VLM star, which corresponds to spectral types late-L/early-T and late-M respectively.

The TNG/Amici low resolution spectra we obtained for these 3 objects, CFHT-Hy-19, 20 and 21 are shown in Fig. 7. The portions of the spectra that are affected by the strongest telluric absorption (around 1.4 and $1.9 \mu \mathrm{m}$ ) are not shown; the portion beyond $2.25 \mu \mathrm{m}$ and shortward of $1 \mu \mathrm{m}$ for CFHT-Hy-21 and CFHT-Hy-20 are also not shown as the signal to noise ratio in the blue end of the spectra is too low and, with the integration time chosen for these objects, the red edge of the spectra is in a non linear regime of the detector due to the high sky background.

The spectra of our targets have been compared with the Amici spectral library of field dwarfs compiled by Testi et al. (2001) and Testi (2004). While the gravity of field dwarfs is expected to be somewhat different from that of Hyades members, at the Amici resolution, the general shape of the spectrum should closely match for similar spectral types. Following this expectation, we have matched our observed spectra with the most similar field dwarf spectra and derived an approximate spectral type for our targets. In Fig. 7 we show as dotted lines the spectra of the closest matching dwarfs together with our target stars. We find an excellent match with dwarfs with spectral types M8, T2 and T1 for CFHT-Hy-19, 20 and 21 respectively; neglecting the possible systematic offset introduced by comparing dwarfs of 

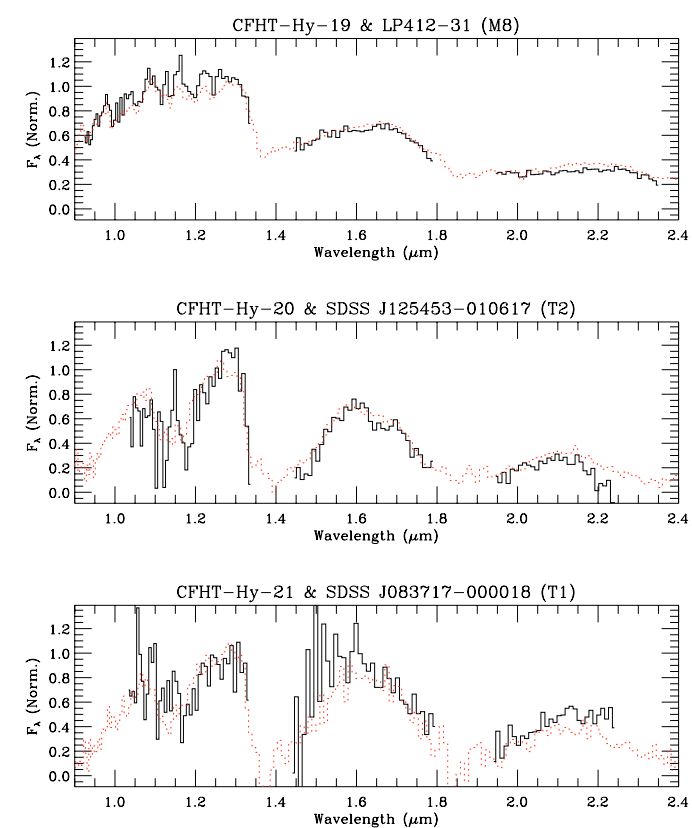

Fig. 7. Near-infrared Amici low resolution spectra of CFHT-Hy-19, 20 and 21 (solid lines from top to bottom). In each panel we also show the closest matching field dwarf spectrum from the low resolution Amici spectral library (Testi et al. 2001; Testi 2004).

different gravity, the uncertainty of our procedure for spectral typing is within one subclass. It is worth pointing out that our near infrared classification of CFHT-Hy-19 agrees with the optical M8 classification of Gizis et al. (1999).

As noted above, the 2 T-dwarfs we report here are strong candidate Hyades members with their photometry and proper motion consistent with membership. Nevertheless, we proceed in estimating the probability that they could be unrelated field $\mathrm{T}$ dwarfs projected onto the Hyades cluster. The 2 observed T-dwarfs have a $K$-band magnitude of 16.08 and 16.59 , respectively. T0-T2 field dwarfs at an age of a few Gyr have an absolute $K$-band magnitude of $\simeq 13.5$ (Chiu et al. 2006). This would then locate the contaminating dwarfs at a distance between approximately 30 and $40 \mathrm{pc}$. Combined with the area covered by our survey, this corresponds to a volume of $65 \mathrm{pc}^{3}$. From the combination of the 2MASS and SDSS DR1 surveys, Metchev et al. (2007) derived an upper limit of $0.9 \times 10^{-3} \mathrm{pc}^{-3}$ on the space density of T0-T2.5 dwarfs in the solar neighborhood. Hence, we would expect $\leq 0.06$ early field $\mathrm{T}$ dwarf to contaminate our survey. An independent empirical estimate is provided by the detection rate of early $\mathrm{T}$ dwarfs in the UKIDSS LAS survey. Chiu et al. (2008) reported the detection of 3 early T-dwarfs up to a distance of $98 \mathrm{pc}$ over $136 \mathrm{deg}^{2}$ in the southern sky. Scaling from their volume to ours, we derive that about 0.06 early field T-dwarfs should contaminate our survey. This further strengthens the likelihood that the 2 candidates we report here are indeed the first BDs and the lowest mass members of the Hyades cluster known to date.

\subsection{The Hyades mass function}

Our survey is complete in the mass range from less than 50 Jupiter masses up to $0.20 M_{\odot}$. We are thus able to derive the cluster's mass function across the stellar-substellar boundary and well into the $\mathrm{BD}$ regime. The mass of the probable members we report in Table 1 was derived using the 600 Myr NextGen
(Baraffe et al. 1998) and Dusty (Chabrier et al. 2000) model isochones, with the transition between NextGen and Dusty taken at $0.08 M_{\odot}\left(T_{\text {eff }}=2500 \mathrm{~K}\right)$. These models provide MassMagnitude relationships in the CFHT $I$ and $z$ system, as well as for the $K$-band. Table 2 lists the mass of the candidates derived from their $I, z$ and $K$ magnitudes $(\mathrm{DM}=3.33 \mathrm{mag})$. Over the mass range $0.05-0.25 M_{\odot}$, mass estimates obtained from the $I$ and $z$ magnitudes agree to within $4 \%$ with no systematic offsets. In contrast, masses derived from $K$ magnitude are usually higher than those derived from the $I$ magnitude by up to $20 \%$ in the stellar domain, and are lower by up to the same amount in the substellar domain. The discrepancy between masses obtained from either visual or near-IR magnitudes presumably results at least in part from model uncertainties but might in some cases be related to the presence of unresolved companions. These uncertainties do not significantly affect the shape of the mass function we derive below and we adopt the masses obtained from the $I$-band magnitude in the following.

In order to build the complete present-day mass function (PDMF) of the Hyades cluster, we combine the results of our survey in the $0.05-0.20 M_{\odot}$ mass range to the $P \& S$ database which covers the mass range from about $0.1 M_{\odot}$ to the most massive Hyades members over the whole cluster area. Since we report only 3 new members (over a total of 18, see Tables 1 and 2) in the mass range from 0.1 to $0.25 M_{\odot}$ that were not previously listed in the $\mathrm{P} \& \mathrm{~S}$ database, we assume that the database is nearly complete down to $0.1 M_{\odot}$ (Reid 1993). Conversely, among the 30 low mass probable members listed in the $\mathrm{P} \& \mathrm{~S}$ database that lie in the area of our survey, 8 are rejected as being non members (see Sect. 3.2). We thus estimate the contamination of the P\&S database by field dwarfs to be about $27 \%$ below $0.3 M_{\odot}$. We assume the P\&S database to be uncontaminated for brighter, better characterized Hyades members. The mass of the probable members from the P\&S database was estimated from their $V$-band magnitude as explained in Appendix C.

Figure 8 show the resulting mass distribution of Hyades probable members over the whole mass range from 0.050 to $3 M_{\odot}$, obtained by combining the results of our survey and the P\&S database. Note that since sub-arcsecond binaries are unresolved in seeing-limited imaging surveys (Patience et al. 1997; Reid \& Gizis 1997), this mass distribution represents the systems mass function. The mass function was built by counting the number of objects in each mass bin and statistically correcting for the contamination of the $\mathrm{P} \& \mathrm{~S}$ database by low mass field dwarfs. In order to account for the different areas covered by the 2 datasets, the number of objects detected in our survey was normalized to the number of objects listed in the P\&S database over the common mass range $0.12-0.19 M_{\odot}$ where the 2 datasets are complete. The normalization factor is 3.4 with, respectively, 10 and 34 objects in our survey and the $P \& S$ database over this mass range. This correction factor is consistent with the extrapolation of the areal coverage of our survey to the whole cluster's area assuming the radial distribution of low mass stars follows a King profile with a core radius of $3 \mathrm{pc}$ and a tidal radius of $10.3 \mathrm{pc}$ (Perryman et al. 1998).

The estimate of the cluster's lower mass function we derive by simply counting the number of BDs and VLM stars detected in our survey and renormalizing to the whole cluster's area implicitely assumes that the radial distribution of the two populations is the same. Alternatively, if the Hyades cluster is fully relaxed, one may expect the core radius to scale with the inverse square root of mass, i.e., that the $\mathrm{BD}$ population is more widely distributed than the low mass stars. A core radius of $3 \mathrm{pc}$ for low mass stars would then translate into $r_{\mathrm{c}} \simeq 7 \mathrm{pc}$ for BDs. 

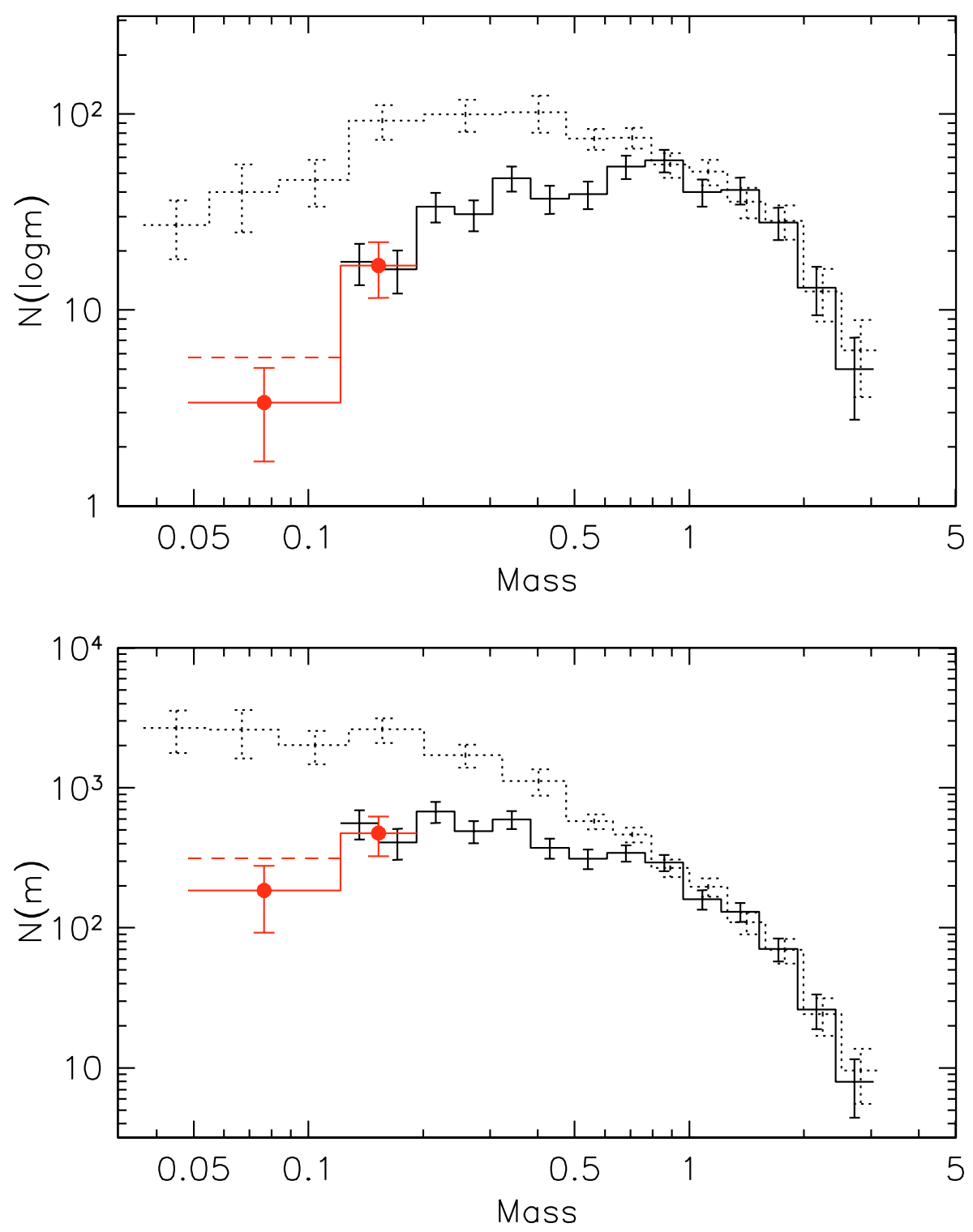

Fig. 8. The mass distribution of Hyades probable members. Upper panel: number of systems per 0.1 logarithmic mass bin. Lower panel: number of systems per unit mass bin. Solid histogram: mass distribution of Hyades systems. The mass distribution derived from our survey is shown as a red histogram in the 2 lowest mass bins $\left(0.048-0.19 M_{\odot}\right)$. It has been normalized to the mass distribution of the P\&S database over the mass range $0.12-0.19 M_{\odot}$ (see text). The dashed histogram in the lowest mass bin $\left(0.048-0.12 M_{\odot}\right)$ assumes a core radius of $7 \mathrm{pc}$ for BDs in the Hyades cluster (see text). Dotted histogram: mass distribution of Pleiades systems from Moraux et al. (2003), normalized to the Hyades PDMF in the mass range $1-3 M_{\odot}$.
Correcting for the different radial distributions of BDs and low mass stars within the 10.3 pc cluster's tidal radius, the actual number of BDs over the cluster's area would then be about $70 \%$ higher than the above estimate. Whenever relevant, this additional correction is taken into account in the discussion below.

Figure 8 compares the resulting Hyades PDMF to that of the Pleiades over the mass range from 0.050 to $3 M_{\odot}$. When expressed as the number of objects per linear mass bin, the mass distribution can be described as a power law over a limited mass range: $\Psi(m)=\mathrm{d} N / \mathrm{d} m \propto m^{-\alpha}$ (Salpeter 1955; Kroupa 2002). In the high mass range $\left(M \geq 1 M_{\odot}\right)$, the Hyades and Pleiades mass functions are found to be similar in shape, with a power law exponent which agrees with a Salpeter slope ( $\alpha=2.35$, Salpeter 1955). However, in the lower mass range, the mass functions of the 2 clusters clearly differ (cf. Fig. 8): while the slope of the power-law MF over the mass range $0.050-0.2 M_{\odot}$ amounts to $\alpha=+0.6$ for the Pleiades (Moraux et al. 2003), we find $\alpha=$ -1.3 for the Hyades over the same mass range (or $\alpha=-0.8$ assuming a $7 \mathrm{pc}$ core radius for Hyades BDs). From the lack of BDs detection in previous surveys, Gizis et al. (1999) found $\alpha=0$ to be an upper limit of the Hyades PDMF below $0.3 M_{\odot}$, which is consistent with our estimate. This points to a strong deficiency of very low mass stars and substellar objects relative to massive stars in the Hyades cluster compared to the MF of Pleiades cluster.

When expressed as the number of objects per logarithmic mass bin, the MF can be approximated by a lognormal functional form:

$\xi(\log m)=\mathrm{d} N / \mathrm{d}(\log m) \propto \exp \left[-\frac{\left(\log m-\log m_{0}\right)^{2}}{2 \sigma^{2}}\right]$

where $m_{0}$ is the peak mass and $\sigma$ the width of the distribution (Miller \& Scalo 1979; Chabrier 2003). A least square fit to the Hyades PDMF over the mass range 0.050 to $3 M_{\odot}$ yields $m_{0} \simeq 0.60 M_{\odot}$ and $\sigma=0.39$ (or $m_{0} \simeq 0.59 M_{\odot}$ and $\sigma=0.40$ assuming a $7 \mathrm{pc}$ core radius for BDs). For the Pleiades it yields $m_{0} \simeq 0.29 M_{\odot}$ and $\sigma=0.47$. The lognormal fits to the mass functions of the 2 clusters are shown in Fig. 9. The deficiency of low mass members in the Hyades cluster's PDMF compared to the MF of the Pleiades cluster is also clearly apparent from these lognormal representations.

Is the present-day Hyades mass function, as we observe it, a dynamically evolved version of the Pleiades mass function? A number of $N$-body simulations have investigated the dynamical 


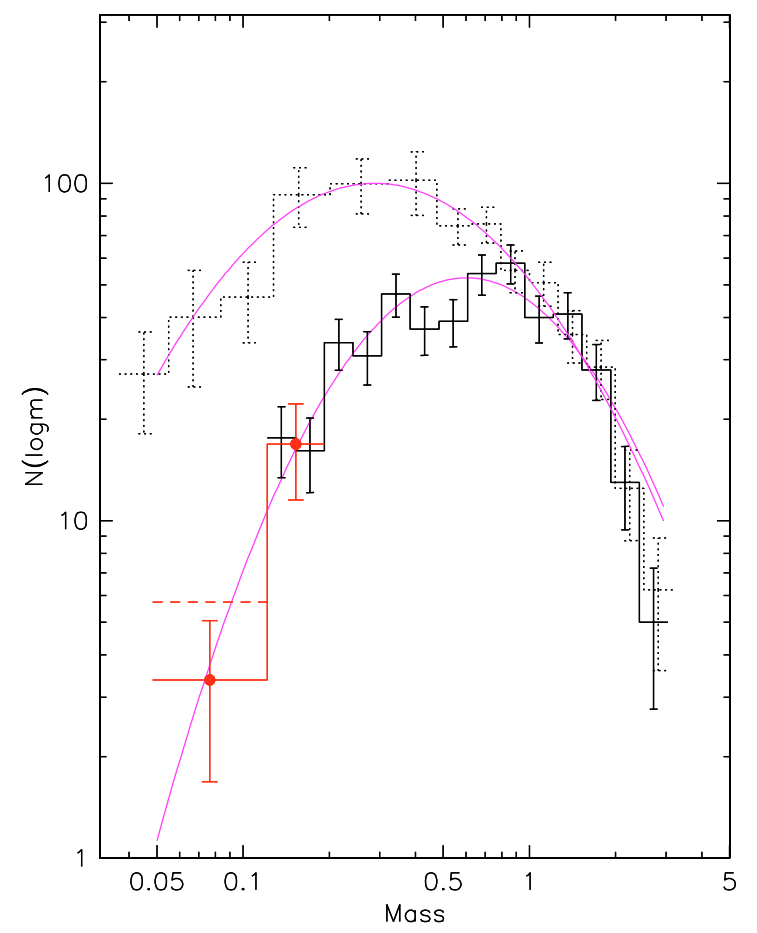

Fig. 9. Lognormal fits to the mass distribution of the Hyades and Pleiades clusters over the mass range $0.050-3.0 M_{\odot}$. The observed mass distributions have been least-square fitted with a lognormal functional form: $\xi(\log m)=k \cdot \exp \left[-\frac{\left(\log m-\log m_{0}\right)^{2}}{2 \sigma^{2}}\right]$ (see text). For the Hyades, we find $k=52.4, m_{0}=0.60 M_{\odot}$, and $\sigma=0.39$, while the best fit to the Pleiades yields $k=100, m_{0}=0.29 M_{\odot}$, and $\sigma=0.47$.

evolution of young stellar clusters and allow us to address this issue. First, our results indicate that the peak stellar mass in the Hyades cluster is $0.6 M_{\odot}$, while it is only $0.3 M_{\odot}$ in the Pleiades cluster. This result is in qualitative agreement with Kroupa's (1995) $N$-body models which predict that the mean cluster mass, at least in the inner few parsecs of the system, linearily increases with time. The increasing peak mass of aging clusters results from cluster members experiencing weak gravitational interactions over a timescale of several $100 \mathrm{Myr}$ thus leading to mass segregation and the preferential evaporation of low mass members (Terlevich 1987).

Numerical models of the dynamical evolution of young open clusters by Adams et al. (2002) further predict that by an age of 625 Myr the Hyades cluster has lost about 2/3 of its initial stellar population and up to $70-90 \%$ of its initial substellar population, depending on the model assumptions. In contrast, at an age of $120 \mathrm{Myr}$, the Pleiades cluster is expected to have lost only $10-15 \%$ of its initial stellar and substellar members (see also de la Fuente Marcos \& de la Fuente Marcos 2000). Assuming that the Hyades cluster is a dynamically-evolved version of the Pleiades cluster, we can estimate from the present-day MF of the 2 clusters the amount of low mass members that have been lost over a timescale of a few $100 \mathrm{Myr}$ on the main sequence. Using the lognormal fits to the Hyades and Pleiades MF obtained above, we find that the Hyades must have lost about $60 \%$ of its initial low mass stellar population $\left(0.08-1.0 M_{\odot}\right)$ and about $95 \%$ of its initial substellar population (or $92 \%$ assuming a 7 pc core radius for BDs in the Hyades). Within modelling and observational uncertainties, these empirical estimates of the preferential evaporation of low mass cluster members compare reasonably well with model predictions.
Hence, the Hyades cluster may well be a dynamicallyevolved version of the Pleiades cluster indeed, as suggested by Kroupa (1995), i.e., both clusters may have had similar initial mass functions down to the substellar domain prior dynamical evolution, as also observed in other young open clusters (de Wit et al. 2006; Moraux et al. 2007). This would suggest that the IMF is not very sensitive to metallicity either, as the Hyades is slightly more metal rich than other young open clusters. Finally, by extrapolating the estimate of the present-day Hyades mass function derived above to the lowest mass BDs down to $0.013 M_{\odot}$ and to the whole cluster area, we predict that the Hyades cluster currently harbors $\sim 10-15$ BDs while it may have counted up to $\sim 150-200$ such objects initially.

With a typical velocity of $\simeq 1 \mathrm{~km} \mathrm{~s}^{-1}$ (de la Fuente Marcos \& de la Fuente Marcos 2000), a fraction of the substellar escapers should now populate the solar neighborhood and be members of the so-called Hyades moving group or "supercluster" (Eggen 1958, 1993; Chereul et al. 1998; Chumak et al. 2005). The space velocity of a few field BDs detected within 20 pc from the Sun indeed seem consistent with their being part of this kinematical stream (Bannister \& Jameson 2007; Zapatero Osorio et al. 2007).

\section{Conclusion}

From a deep, wide-field survey of the central region of the Hyades cluster, we identified new very low-mass cluster members, including 2 T-dwarfs with a mass of $\simeq 50$ Jupiter masses. The comparison of the Hyades lower mass function with that of younger clusters indicates that the cluster is strongly depleted in very low mass objects at an age of $625 \mathrm{Myr}$. We thus estimate a total of about 15 BDs in the present-day Hyades cluster compared to about 500 stars. This depletion appears to result from the preferential evaporation of the lowest mass cluster members over a timescale of several $100 \mathrm{Myr}$, as predicted by $N$-body models of the dynamical evolution of young clusters. A fraction of the substellar escapers should populate the solar neighborhood, and may be detected as members of the Hyades moving group from large scale surveys.

Acknowledgements. We thank the TNG staff for the support in preparing and executing the service observing observations. This research benefited from financial assistance from the European Union Research Training Network "The Formation and Evolution of Young Stellar Clusters" (RTN1-1999-00436). G.M. acknowledges financial support by the DFG under Grant ME2061/3-1. This research has made use of the SIMBAD database, operated at CDS, Strasbourg, France, and of the IRAF package distributed by the NOAO.

\section{References}

Adams, T., Davies, M. B., Jameson, R. F., \& Scally, A. 2002, MNRAS, 333, 547 Allen, P. R., Koerner, D. W., Reid, I. N., \& Trilling, D. E. 2005, ApJ, 625, 385 André, P., Motte, F., \& Bacmann, A. 1999, ApJ, 513, L57

Baffa, C., Comoretto, G., Gennari, S., et al. 2001, A\&A, 378, 722 Bannister, N. P., \& Jameson, R. F. 2007, MNRAS, 378, L24

Barrado y Navascués, D., Stauffer, J. R., Bouvier, J., Jayawardhana, R., \& Cuillandre, J.-C. 2004, ApJ, 610, 1064

Baraffe, I., Chabrier, G., Allard, F., \& Hauschildt, P. H. 1998, A\&A, 337, 403 Bate, M. R., \& Bonnell, I. A. 2005, MNRAS, 356, 1201

Bertin, E., \& Arnouts, S. 1996, A\&AS, 117, 393

Bouvier, J., Moraux, E., \& Stauffer, J. 2005, The Initial Mass Function 50 Years Later, 327, 61

Bouvier, J., Stauffer, J. R., Martin, E. L., et al. 1998, A\&A, 336, 490

Bryja, C., Humphreys, R. M., \& Jones, T. J. 1994, AJ, 107, 246

Caballero, J. A., Béjar, V. J. S., Rebolo, R., et al. 2007, A\&A, 470, 903

Chabrier, G. 2002, ApJ, 567, 304

Chabrier, G. 2003, PASP, 115, 763 
Chabrier, G., Baraffe, I., Allard, F., \& Hauschildt, P. 2000, ApJ, 542, 464

Chereul, E., Creze, M., \& Bienayme, O. 1998, A\&A, 340, 384

Chiu, K., Fan, X., Leggett, S. K., et al. 2006, AJ, 131, 2722

Chiu, K., Liu, M. C., Jiang, L., et al. 2008, MNRAS, 385, L53

Chumak, Y. O., Rastorguev, A. S., \& Aarseth, S. J. 2005, Astron. Lett., 31, 308

Cruz, K. L., Reid, I. N., Kirkpatrick, J. D., et al. 2007, AJ, 133, 439

Cuillandre, J., Luppino, G. A., Starr, B. M., \& Isani, S. 2000, Proc. SPIE, 4008, 1010

de La Fuente Marcos, R., \& de La Fuente Marcos, C. 2000, Ap\&SS, 271, 127

de Wit, W. J., Bouvier, J., Palla, F., et al. 2006, A\&A, 448, 189

Dahn, C. C., Harris, H. C., Verba, F. J., et al. 2002, AJ, 124, 1170

Dobbie, P. D., Kenyon, F., Jameson, R. F., et al. 2002, MNRAS, 329, 543

Eggen, O. J. 1958, MNRAS, 118, 65

Eggen, O. J. 1993, AJ, 106, 1885

Frink, S., Röser, S., Neuhäuser, R., \& Sterzik, M. F. 1997, A\&A, 325, 613

Gizis, J. E., Reid, I. N., \& Monet, D. G. 1999, AJ, 118, 997

Hanson, R. B. 1975, AJ, 80, 379

Hunt, L. K., Mannucci, F., Testi, L., et al. 1998, AJ, 115, 2594

Jappsen, A.-K., Klessen, R. S., Larson, R. B., Li, Y., \& Mac Low, M.-M. 2005, A\&A, 435, 611

Kroupa, P. 1995, MNRAS, 277, 1522

Kroupa, P. 2002, Science, 295, 82

Landolt, A. U. 1992, AJ, 104, 340

Larson, R. B. 2005, MNRAS, 359, 211

Lee, C.-F., Mundy, L. G., Stone, J. M., \& Ostriker, E. C. 2002, ApJ, 576, 294

Leggett, S. K., \& Hawkins, M. R. S. 1988, MNRAS, 234, 1065

Leggett, S. K., \& Hawkins, M. R. S. 1989, MNRAS, 238, 145

Leggett, S. K., Harris, H. C., \& Dahn, C. C. 1994, AJ, 108, 944

Lucas, P. W., Weights, D. J., Roche, P. F., \& Riddick, F. C. 2006, MNRAS, 373, L60

Luhman, K. L., Stauffer, J. R., Muench, A. A., et al. 2003, ApJ, 593, 1093

Luyten, W. J., Hill, G., \& Morris, S. 1981, Proper Motion Survey, Minneapolis, Univ. of Minesota

Magnier, E. A., \& Cuillandre, J.-C. 2004, PASP, 116, 449
Metchev, S. A., Kirkpatrick, J. D., Berriman, G. B., \& Looper, D. 2007, [arXiv: 0710.4157]

Miller, G. E., \& Scalo, J. M. 1979, ApJS, 41, 513

Mink, D. 2006, Astronomical Data Analysis Software and Systems XV, 351, 204

Moraux, E., Bouvier, J., Stauffer, J. R., \& Cuillandre, J.-C. 2003, A\&A, 400, 891

Moraux, E., Bouvier, J., \& Clarke, C. 2005, Mem. Soc. Astron. Ital., 76, 265

Moraux, E., Bouvier, J., Stauffer, J. R., Barrado y Navascués, D., \& Cuillandre, J.-C. 2007, A\&A, 471, 499

Oliva, E. 2003, Mem. Sc. Astr. It., 74, 118

Onishi, T., Mizuno, A., Kawamura, A., Tachihara, K., \& Fukui, Y. 2002, ApJ, 575,950

Padoan, P., \& Nordlund, Å. 2002, ApJ, 576, 870

Patience, J., Ghez, A. M., Reid, I. N., Weinberger, A. J., \& Matthews, K. 1997, BAAS, 29, 1339

Perryman, M. A. C., Brown, A. G. A., Lebreton, Y., et al. 1998, A\&A, 331, 81

Pinsonneault, M. H., Terndrup, D. M., Hanson, R. B., \& Stauffer, J. R. 2004, ApJ, 600, 946

Portegies Zwart, S. F., McMillan, S. L. W., Hut, P., \& Makino, J. 2001, MNRAS, 321,199

Reid, N. 1992, MNRAS, 257, 257

Reid, N. 1993, MNRAS, 265, 785

Reid, I. N., \& Gizis, J. E. 1997, AJ, 114, 1992

Reid, I. N., \& Hawley, S. L. 1999, AJ, 117, 343

Reid, I. N., \& Mahoney, S. 2000, MNRAS, 316, 827

Reid, I. N., Kirkpatrick, J. D., Liebert, J., et al. 1999, ApJ, 521, 613

Robin, A. C., Reylé, C., Derrière, S., \& Picaud, S. 2003, A\&A, 409, 523 Salpeter, E. E. 1955, ApJ, 121, 161

Siegler, N., Close, L. M., Mamajek, E. E., \& Freed, M. 2003, ApJ, 598, 1265

Starr, B. M., Doyon, R., Beuzit, J.-L., et al. 2000, Proc. SPIE, 4008, 999

Terlevich, E. 1987, MNRAS, 224, 193

Testi, L. 2004, Mem. Sc. Astr. It., 75, 89

Testi, L., D'Antona, F., Ghinassi, F., et al. 2001, ApJ, 552, L147

van Altena, W. F. 1969, AJ, 74, 2

Zapatero Osorio, M. R., Martín, E. L., Béjar, V. J. S., et al. 2007, ApJ, 666, 1205 
Table A.1. CFHT 12K fields. Date of observation, coordinates of mosaic center, seeing $F W H M$.

\begin{tabular}{|c|c|c|c|c|}
\hline$\overline{\text { Field }}$ & 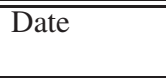 & $\begin{array}{l}\text { RA } \\
(2000)\end{array}$ & $\begin{array}{l}\text { Dec } \\
(2000)\end{array}$ & $\begin{array}{l}\text { Seeing } \\
1\end{array}$ \\
\hline HYADES-A1 & $2002-10-01$ & $4: 26: 59.27$ & $15: 56: 02.3$ & 0.8 \\
\hline HYADES-A2 & 2002-10-01 & $4: 26: 40.56$ & $16: 22: 44.0$ & 0.7 \\
\hline HYADES-A3 & 2002-10-01 & $4: 23: 45.80$ & $16: 22: 33.0$ & 0.7 \\
\hline HYADES-A4 & 2002-10-01 & $4: 23: 58.76$ & $15: 56: 41.9$ & 0.6 \\
\hline HYADES-A5 & $2002-10-01$ & 4:30:15.06 & $15: 55: 45.5$ & 0.6 \\
\hline HYADES-A6 & $2002-10-03$ & 4:30:01.01 & $16: 26: 36.0$ & 0.8 \\
\hline HYADES-A7 & $2002-10-03$ & $4: 28: 30.80$ & $16: 49: 23.1$ & 0.6 \\
\hline HYADES-A8 & $2002-10-03$ & $4: 25: 00.50$ & $16: 48: 19.9$ & 0.6 \\
\hline HYADES-A9 & $2002-10-03$ & $4: 28: 30.40$ & $15: 30: 45.2$ & 0.6 \\
\hline HYADES-A10 & 2002-11-05 & $4: 24: 31.74$ & $15: 29: 27.0$ & 0.7 \\
\hline HYADES-A11 & 2002-10-02 & $4: 24: 20.31$ & $15: 03: 30.2$ & 0.8 \\
\hline HYADES-A12 & 2002-11-05 & $4: 27: 11.04$ & $15: 03: 45.6$ & 0.8 \\
\hline HYADES-A13 & 2002-11-05 & $4: 29: 30.52$ & 15:03:38.6 & 0.8 \\
\hline HYADES-B1 & 2002-11-05 & $4: 26: 00.47$ & $17: 24: 35.5$ & 0.8 \\
\hline HYADES-B2 & $2002-11-06$ & $4: 27: 31.22$ & $17: 51: 16.7$ & 0.7 \\
\hline HYADES-B3 & $2002-11-06$ & $4: 22: 31.05$ & $17: 04: 31.0$ & 0.7 \\
\hline HYADES-B4 & 2002-11-06 & $4: 21: 31.11$ & $16: 31: 38.0$ & 0.8 \\
\hline HYADES-B5 & 2002-11-06 & $4: 21: 11.31$ & $16: 05: 48.4$ & 0.6 \\
\hline HYADES-B6 & 2002-11-06 & $4: 21: 40.84$ & $15: 39: 44.6$ & 0.6 \\
\hline HYADES-B7 & 2002-11-07 & $4: 21: 41.61$ & $15: 21: 27.5$ & 0.7 \\
\hline HYADES-B8 & 2002-11-07 & $4: 21: 41.14$ & $14: 39: 40.4$ & 0.8 \\
\hline HYADES-B9 & 2002-11-07 & $4: 24: 25.86$ & $14: 29: 41.7$ & 0.7 \\
\hline HYADES-B10 & 2002-11-07 & $4: 24: 25.96$ & $14: 04: 43.5$ & 0.7 \\
\hline HYADES-B11 & 2002-11-07 & $4: 27: 11.16$ & $14: 19: 46.3$ & 0.6 \\
\hline HYADES-B12 & 2002-11-08 & 4:30:00.81 & $14: 34: 46.3$ & 0.7 \\
\hline HYADES-B13 & 2002-11-08 & 4:32:09.73 & $14: 49: 39.8$ & 0.6 \\
\hline HYADES-B14 & 2002-11-08 & 4:32:00.09 & $15: 24: 44.2$ & 0.7 \\
\hline HYADES-B15 & 2002-11-09 & $4: 33: 31.42$ & $15: 49: 37.3$ & 0.7 \\
\hline HYADES-B16 & 2002-11-09 & $4: 32: 20.49$ & $16: 19: 41.0$ & 0.6 \\
\hline HYADES-B17 & $2002-11-10$ & $4: 31: 20.45$ & $16: 51: 34.8$ & 0.8 \\
\hline HYADES-B18 & $2002-11-12$ & 4:31:00.92 & $17: 16: 33.2$ & 0.6 \\
\hline HYADES-C01 & 2002-11-08 & 4:20:30.91 & $17: 29: 29.6$ & 1.0 \\
\hline HYADES-C02 & $2003-01-26$ & 4:23:19.97 & $17: 54: 38.1$ & 0.7 \\
\hline HYADES-C03 & 2003-01-26 & $4: 25: 20.42$ & $18: 16: 46.3$ & 0.7 \\
\hline HYADES-C04 & 2003-01-27 & 4:23:00.30 & $18: 41: 34.7$ & 0.5 \\
\hline HYADES-C0 $5^{\dagger}$ & 2003-01-28 & 4:20:29.31 & $17: 54: 29.9$ & 0.8 \\
\hline HYADES-C06 & $2002-12-27$ & 4:19:40.61 & $18: 21: 26.5$ & 0.6 \\
\hline HYADES-C07 & 2003-01-28 & $4: 19: 50.05$ & $17: 04: 46.1$ & 0.6 \\
\hline HYADES-C08 & 2003-01-28 & $4: 18: 10.61$ & $16: 39: 40.4$ & 0.7 \\
\hline HYADES-C09 & 2002-09-07 & 4:17:06.06 & $17: 02: 16.8$ & 0.6 \\
\hline HYADES-C10 & 2002-09-07 & $4: 17: 40.56$ & $17: 27: 27.1$ & 0.6 \\
\hline HYADES-C13 & $2002-12-04$ & $4: 27: 15.45$ & $13: 54: 16.5$ & 0.9 \\
\hline HYADES-C14 & 2002-12-04 & 4:30:00.29 & 14:09:24.2 & 0.8 \\
\hline HYADES-C15 & 2002-12-04 & $4: 32: 45.17$ & $14: 23: 26.2$ & 0.7 \\
\hline HYADES-C16 & 2002-12-04 & $4: 35: 45.31$ & $14: 59: 29.8$ & 0.8 \\
\hline HYADES-C17 & 2002-12-04 & 4:36:09.91 & $15: 34: 27.0$ & 0.6 \\
\hline HYADES-C18 & 2002-12-04 & 4:26:19.49 & $13: 29: 23.0$ & 0.7 \\
\hline HYADES-C19 & $2002-12-06$ & 4:29:00.53 & $13: 37: 29.3$ & 0.6 \\
\hline HYADES-C20 & $2002-12-04$ & 4:32:59.91 & $13: 57: 22.3$ & 0.8 \\
\hline HYADES-C21 & $2002-12-05$ & $4: 35: 29.56$ & $14: 33: 15.5$ & 0.6 \\
\hline HYADES-C22 & $2002-12-06$ & 4:30:29.97 & $13: 12: 25.9$ & 0.7 \\
\hline HYADES-C23 & $2002-12-06$ & $4: 33: 15.01$ & $13: 32: 31.7$ & 0.8 \\
\hline HYADES-C24 & $2002-12-06$ & $4: 35: 28.15$ & 14:07:29.3 & 0.5 \\
\hline
\end{tabular}

$\dagger$ No short exposure was obtained for this field.

\section{Appendix A: Coordinates of CFHT12K Hyades fields}

\section{Appendix B: Finding charts}

We provide here finding charts for the 2 detected T-dwarfs in the Hyades cluster. The finding charts are a subset of the CFHT12K I-band images.

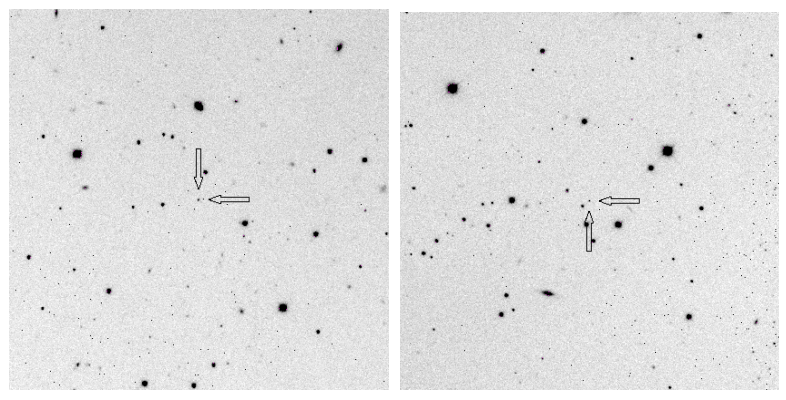

Fig. B.1. $I$-band finding charts for CFHT-Hy-20 (left) and CFHT-Hy-21 $\left(\right.$ right). The size is $3 \times 3^{\prime}$, North is up, East is left.

\section{Appendix C: Estimating the mass of probable Hyades members in the Prosser \& Stauffer database}

The Prosser \& Stauffer database lists 521 probable Hyades members. In order to combine this database with our dataset and build the complete mass function for the cluster, we derived the mass of the $\mathrm{P} \& \mathrm{~S}$ database probable members from their $V$-band magnitude (with $\mathrm{DM}=3.33$ ). For objects with a mass larger than $0.6 M_{\odot}$, we used the Mass- $M_{V}$ relationship derived by Pinsonneault et al. (2004) for the Hyades. The Mass$M_{V}$ relationship spans the mass range from 0.636 to $2.235 M_{\odot}$, corresponding to $M_{V}$ from 1.10 to 8.50 (and $V$ from 4.43 to 11.83). For objects more massive than $2.2 M_{\odot}$, we linearily extrapolated Pinsonneault et al.'s (2004) Mass- $M_{V}$ relationship, up to the most massive Hyades probable member listed in the P\&S database with a mass of $2.67 M_{\odot}$.

For objects less massive than $0.6 M_{\odot}$, we used the massmagnitude relationships provided by the Lyon models (Baraffe et al. 1998; Chabrier et al. 2000). Down to $0.08 M_{\odot}$, we used the NextGen $600 \mathrm{Myr}$ isochrone, while for lower mass objects we used the Dusty $600 \mathrm{Myr}$ isochrone. The NextGen to Dusty transition is thus taken to arise at $0.08 M_{\odot}$, corresponding to an effective temperature of $2500 \mathrm{~K}$. It is usually acknowledged that the Lyon models are not totally reliable in the $V$-band, due to missing opacity sources. Hence, we prefer to derive the mass of the candidates from their I-band magnitude, since models are believed to be more reliable at this wavelength. However, while all probable members listed in the $\mathrm{P} \& \mathrm{~S}$ database have a measured $V$-band magnitude, only a fraction of them have a known $I$-band magnitude. We thus proceeded in 2 steps.

We first estimated the masses of a subsample of 356 candidates in the database that have both $V$ - and $I$-magnitudes. Two estimates of the mass is obtained for each object, one from the Mass- $M_{V}$ isochrone, the other from the Mass- $M_{I}$ isochrone. Masses derived in both ways are compared in Fig. C.1 over the mass range from 0.07 to $1.2 M_{\odot}$. It is seen that masses derived from $M_{V}$ tends to be systematically lower than those derived from $M_{I}$ by up to $20 \%$ at masses less than $0.6 M_{\odot}$, while they become slightly larger, by up to $5 \%$, for more massive objects. We thus used these mass estimates to compute the correction to be applied to the $V$-band mass in order to convert it to an $I$-band mass. The correction is shown as the spline fit interpolation in Fig. C.1. We then simply proceed by deriving the mass from the $V$ magnitudes of all the low mass P\&S database probable members and applying the correction computed above to transform it to an $I$-band model mass.

The resulting Mass- $M_{V}$ relationship we use over the whole mass range of Hyades probable members is shown in Fig. C.1 and is listed in Table C.1. This Mass- $M_{V}$ relationship combines 
J. Bouvier et al.: Brown dwarfs and very low mass stars in the Hyades cluster: a dynamically evolved mass function, Online Material $p 2$
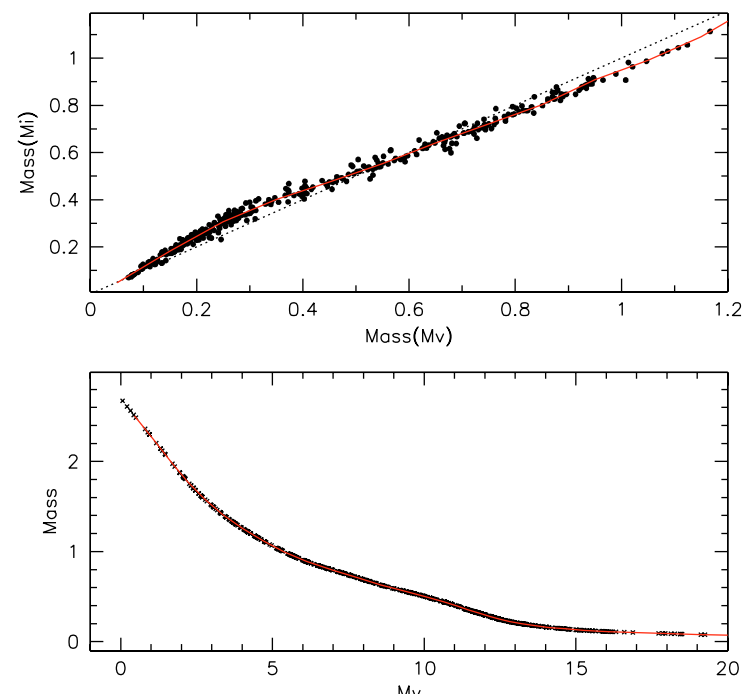

Fig. C.1. Top: comparison of the masses estimated from the Lyon models using either the Mass- $M_{V}\left(x\right.$-axis) or the Mass- $M_{I}$ ( $y$-axis) $600 \mathrm{Myr}$ isochrone. The dotted line shows the locus of equal masses while the solid line is a spline interpolation of the data. Bottom: the Mass- $M_{V}$ relationship (solid line) used to derive mass from the $V$-band magnitudes of probable Hyades members from the Prosser \& Stauffer database (crosses). Above $0.6 M_{\odot}$, the relationship is that of Pinsonneault et al. (2004), below $0.6 M_{\odot}$ it is derived from the Lyon models (see text).

Table C.1. The Hyades $M_{V}$-Mass relationship used in this study.

\begin{tabular}{llllllll}
\hline \hline$M_{V}$ & $\begin{array}{l}\text { Mass } \\
\left(M_{\odot}\right)\end{array}$ & $M_{V}$ & $\begin{array}{l}\text { Mass } \\
\left(M_{\odot}\right)\end{array}$ & $M_{V}$ & $\begin{array}{l}\text { Mass } \\
\left(M_{\odot}\right)\end{array}$ & $M_{V}$ & $\begin{array}{l}\text { Mass } \\
\left(M_{\odot}\right)\end{array}$ \\
\hline 23.25 & 0.050 & 14.68 & 0.141 & 11.08 & 0.397 & 4.69 & 1.119 \\
21.66 & 0.056 & 14.11 & 0.158 & 10.61 & 0.446 & 4.01 & 1.256 \\
21.00 & 0.063 & 13.65 & 0.177 & 10.04 & 0.500 & 3.37 & 1.409 \\
20.00 & 0.071 & 13.22 & 0.199 & 9.34 & 0.561 & 2.77 & 1.581 \\
18.99 & 0.079 & 12.81 & 0.223 & 8.56 & 0.630 & 2.21 & 1.774 \\
18.06 & 0.089 & 12.46 & 0.251 & 7.84 & 0.706 & 1.67 & 1.991 \\
17.03 & 0.100 & 12.16 & 0.281 & 7.01 & 0.792 & 1.10 & 2.233 \\
16.06 & 0.111 & 11.82 & 0.316 & 6.12 & 0.889 & 0.45 & 2.506 \\
15.26 & 0.126 & 11.44 & 0.354 & 5.38 & 0.998 & & \\
\hline
\end{tabular}

Pinsonneault et al.'s (2004) one for masses larger than $0.6 M_{\odot}$ and the Lyon models for lower mass objects.

\section{Appendix D: Hyades probable non-members}

We provide here a list of optically-selected Hyades candidates from the $(I, I-z)$ CMD which turn out to be non members based on follow up $K$-band photometry and proper motion measurement. 
J. Bouvier et al.: Brown dwarfs and very low mass stars in the Hyades cluster: a dynamically evolved mass function, Online Material p 3

Table D.1. Hyades probable non-members: candidate members selected from the $(I-, I-z)$ CMD but whose infrared colors and/or proper motion is inconsistent with membership.

\begin{tabular}{|c|c|c|c|c|c|c|c|c|}
\hline Name & $\overline{\mathrm{RA}(2000)}$ & $\overline{\operatorname{Dec}(2000)}$ & $I$ & $\overline{I-z}$ & $\overline{I-K}$ & $\begin{array}{l}\mu_{\alpha \cos \delta} \\
\left(\mathrm{mas}^{-} \mathrm{yr}^{-}\right.\end{array}$ & $\begin{array}{l}\mu_{\delta} \\
\left.{ }^{1}\right)\end{array}$ & Other name \\
\hline HA7Ic02-222s & 42814.2 & 16580 & 12.96 & 0.46 & 2.22 & 26 & -5 & VA 477 \\
\hline HA2Ic07-156s & 42556.4 & 16138 & 13.58 & 0.52 & 2.35 & 7 & -21 & \\
\hline $\mathrm{HC} 13 \mathrm{c} 03-570 \mathrm{~s}$ & 42718.6 & 13558 & 13.6 & 0.5 & 2.37 & -46 & -12 & \\
\hline HB18c03-31s & 4313.5 & 172939 & 13.76 & 0.62 & 2.52 & -37 & -59 & \\
\hline HA3Ic03-197s & 42357 & 163244 & 13.83 & 0.53 & 2.33 & 1 & 0 & \\
\hline HA2Ic07-287s & 42543.6 & 161621 & 13.87 & 0.56 & 2.44 & 24 & -37 & \\
\hline HA1Ic07-119s & 42625.8 & 15453 & 13.92 & 0.72 & 3.13 & -25 & 17 & \\
\hline HB1Ic04-70s & 42653.3 & 173648 & 13.94 & 0.53 & 2.25 & 47 & -4 & \\
\hline HA5Ic02-407s & 42959.1 & 16259 & 14.08 & 0.54 & 2.31 & -117 & -235 & VA 562, LP $415-143$ \\
\hline HA9Ic06-243s & 4274.7 & 152429 & 14.14 & 0.54 & 2.32 & -43 & -14 & \\
\hline $\mathrm{HB} 18 \mathrm{c} 02-680 \mathrm{~s}$ & 43041.8 & 171943 & 14.18 & 0.74 & 2.83 & 26 & 8 & \\
\hline $\mathrm{HC} 02 \mathrm{c} 09-531 \mathrm{~s}$ & 42325.8 & 175344 & 14.25 & 0.55 & 2.51 & 149 & -139 & \\
\hline HB2Ic01-63s & 42640.7 & 18417 & 14.27 & 0.6 & 2.51 & 59 & -132 & \\
\hline HB18c09-393s & 43112.4 & 17942 & 14.3 & 0.68 & 2.73 & 50 & -87 & \\
\hline HB11c08-273s & 42647.9 & 141158 & 14.36 & 0.57 & 2.37 & 69 & -79 & \\
\hline HC14c01-191s & 42919.9 & 141936 & 14.53 & 0.67 & 2.67 & 5 & 6 & \\
\hline $\mathrm{HC} 09 \mathrm{c} 11-32 \mathrm{~s}$ & 41821.4 & 165134 & 14.58 & 0.6 & 2.61 & -27 & -64 & \\
\hline HB3Ic09-301s & 42239 & 165750 & 14.74 & 0.59 & 2.42 & 9 & -24 & \\
\hline HB3Ic10-505s & 42327.7 & 17227 & 14.78 & 0.63 & 2.5 & 9 & -1 & \\
\hline HA9Ic09-44s & 42858.6 & 151737 & 14.86 & 0.69 & 2.76 & 77 & -104 & \\
\hline HB7Ic04-145s & 42214.1 & 153052 & 15.36 & 0.78 & 4.03 & 25 & -6 & h4046b \\
\hline HB1Ic08-643s & 42539.3 & 17232 & 15.56 & 0.72 & 2.66 & 20 & -5 & \\
\hline HB2Ic01-214s & 42632.1 & 18027 & 15.56 & 0.75 & 2.9 & -9 & 8 & \\
\hline HA10c04-89s & 42514.2 & 15418 & 17.42 & 1.01 & 3.94 & 75 & -120 & \\
\hline HB1Ic01-3861 & 42523.1 & 173514 & 17.54 & 1.07 & 3.79 & -2 & -11 & LH $0422+17$, LH 994 \\
\hline $\mathrm{HC} 21 \mathrm{c} 04-2512 \mathrm{l}$ & 4363.1 & 143537 & 18.21 & 1.01 & 3.96 & 21 & -52 & \\
\hline HB $13 \mathrm{c} 09-22 \mathrm{~s}$ & 43225.8 & 143544 & 18.77 & 1.06 & 3.41 & 15 & -49 & \\
\hline HC10c00-1861 & 41621.6 & 174248 & 18.84 & 1.03 & 3.48 & 28 & -17 & \\
\hline HC09c10-13081 & 41748.4 & 165559 & 19.05 & 1.13 & 2.8 & 15 & 9 & \\
\hline $\mathrm{HC} 24 \mathrm{c} 02-487 \mathrm{~s}$ & 43510.4 & 141251 & 19.13 & 1.11 & 4.04 & 32 & -13 & \\
\hline HB4Ic04-257s & 4224.5 & 163944 & 19.18 & 1.03 & 3.95 & 27 & -15 & \\
\hline HC09c10-26501 & 41741.1 & 17114 & 19.21 & 1.19 & 3.73 & 22 & -20 & \\
\hline HB7Ic11-463s & 4234.7 & 151857 & 19.38 & 1.08 & 4.18 & 40 & -31 & h7334b \\
\hline HB15c00-4l & 4329.9 & 16328 & 19.67 & 1.07 & 3.85 & -7 & 7 & \\
\hline HA2Ic04-4741 & 42722 & 163413 & 19.76 & 1.07 & 3.8 & -17 & -2 & \\
\hline HC21c07-1526l & 43457.4 & 14288 & 19.8 & 1.09 & 5.33 & -5 & -23 & \\
\hline $\mathrm{HC} 03 \mathrm{c} 03-516 \mathrm{~s}$ & 42527.2 & 182421 & 19.84 & 1.2 & 5.23 & -14 & -71 & \\
\hline $\mathrm{HC} 22 \mathrm{c} 00-15081$ & 42919.3 & 131721 & 19.85 & 1.12 & 3.96 & -31 & 13 & \\
\hline HB6Ic01-25s & 42049.5 & 155333 & 19.86 & 1.06 & 3.84 & 24 & -15 & \\
\hline HA9Ic02-41s & 42830.1 & 15437 & 19.89 & 1.1 & 4.15 & 15 & -60 & \\
\hline HB8Ic08-1711 & 42117.2 & 142654 & 19.9 & 1.08 & 3.84 & -9 & -8 & \\
\hline HA12c07-1011 ${ }^{\dagger}$ & 42612.8 & 145029 & 20 & 1.07 & 3.53 & 90 & -19 & \\
\hline HB12c04-5941 & 43035.3 & 144532 & 20.11 & 1.08 & 4.34 & 3 & -49 & \\
\hline HB12c10-13271 & 43052 & 142641 & 20.19 & 1.1 & 3.73 & -11 & 2 & \\
\hline HB2Ic07-2871 & 42650.2 & 173930 & 20.28 & 1.11 & 4.41 & -13 & -55 & \\
\hline HC15c00-5301 & 43128.7 & 143513 & 20.29 & 1.15 & 3.98 & -14 & 0 & \\
\hline HA1Ic07-9301 & 4265.2 & 154926 & 20.3 & 1.12 & 4.23 & 46 & -30 & \\
\hline $\mathrm{HC} 15 \mathrm{c} 09-2131$ & 4338.9 & 141040 & 20.45 & 1.11 & 4.07 & 13 & -26 & \\
\hline HB7Ic04-12731 & 42219.2 & 152554 & 20.78 & 1.12 & 5.05 & -11 & 9 & \\
\hline HC21c11-741 & 43646.1 & 141937 & 20.79 & 1.13 & 3.91 & 21 & 10 & \\
\hline HA9Ic02-2661 & 42827.5 & 154223 & 20.89 & 1.12 & 4.81 & 2 & -1 & \\
\hline HA10c04-6411 & 42524 & 153941 & 20.99 & 1.12 & 3.43 & 20 & -50 & \\
\hline HA3Ic01-11811 & 4238.3 & 162930 & 21.02 & 1.13 & 4.23 & 2 & 13 & \\
\hline HB17c07-1416l & 43029.7 & 164445 & 21.09 & 1.12 & 4.62 & 32 & 49 & \\
\hline HC10c01-871 & 41650.2 & 174255 & 21.17 & 1.12 & 4.2 & 0 & 13 & \\
\hline HA1Ic09-4601 & 42716.5 & 154533 & 21.19 & 1.19 & 4.41 & -26 & -13 & \\
\hline HC14c01-6071 & 42922.7 & 141910 & 21.36 & 1.2 & 4.69 & -25 & -21 & \\
\hline HB7Ic10-12891 & 42235.3 & 151524 & 21.38 & 1.18 & 4.43 & -5 & 13 & \\
\hline HB2Ic08-9921 & 4271.8 & 174454 & 21.42 & 1.18 & 4.76 & -32 & -37 & \\
\hline HB13c11-245l & 43325.4 & 143628 & 21.5 & 1.18 & 4.5 & 6 & -16 & \\
\hline HB3Ic11-684l & 42346.7 & 165339 & 21.51 & 1.2 & 4.19 & 13 & -16 & \\
\hline HB17c06-9991 & 43013.2 & 16439 & 21.55 & 1.19 & 5.46 & 17 & -7 & \\
\hline $\mathrm{HC} 03 \mathrm{c} 04-2872 \mathrm{l}$ & 4266.5 & 182025 & 21.55 & 1.18 & 4.43 & 20 & -9 & \\
\hline
\end{tabular}


Table D.1. continued.

\begin{tabular}{|c|c|c|c|c|c|c|c|c|}
\hline Name & 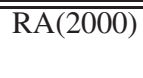 & 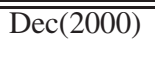 & $\overline{\overline{I I}}$ & $\overline{\overline{I-z}}$ & $\overline{I-K}$ & $\begin{array}{l}\mu_{\alpha \cos \delta} \\
\left(\mathrm{mas}_{\mathrm{yr}}^{-}\right.\end{array}$ & $\begin{array}{l}\mu_{\delta} \\
1)\end{array}$ & "Other name \\
\hline HB12c02-1504l & 42945.8 & 143910 & 21.63 & 1.17 & 4.14 & 5 & -30 & \\
\hline HB13c07-15641 & 43115.5 & 144356 & 21.69 & 1.3 & 5.1 & -5 & -2 & \\
\hline HB1Ic03-15361 & 42620.8 & 172822 & 21.7 & 1.24 & 4.07 & 48 & 52 & \\
\hline HC06c07-19931 & 4196.1 & 182115 & 21.87 & 1.23 & 5.19 & 10 & -34 & \\
\hline HA $11 \mathrm{c} 07-8031^{1}$ & 42342 & 145843 & 21.89 & 1.15 & & & & \\
\hline HC10c10-17961 ${ }^{1}$ & 41827 & 172439 & 21.93 & 1.19 & & & & \\
\hline HB9Ic07-2341 & 42327 & 141723 & 21.94 & 1.23 & 5.04 & 79 & 72 & \\
\hline $\mathrm{HC} 06 \mathrm{c} 02-152 \mathrm{l}^{1}$ & 41922 & 183442 & 22.00 & 1.17 & & & & \\
\hline $\mathrm{HC} 19 \mathrm{c} 03-2076 \mathrm{l}^{1}$ & 42921 & 134010 & 22.10 & 1.18 & & & & \\
\hline $\mathrm{HC} 02 \mathrm{c} 07-5781$ & 42228 & 174355 & 22.13 & 1.22 & 5.32 & 36 & -26 & \\
\hline HB17c08-21771 ${ }^{1}$ & 43118 & 164934 & 22.16 & 1.17 & & & & \\
\hline HA7Ic07-1801 ${ }^{1}$ & 42734 & 163711 & 22.21 & 1.19 & & & & \\
\hline HC13c00-5201 & 42552.9 & 14611 & 22.28 & 1.23 & 4.48 & 1 & 46 & \\
\hline HА6Ic01-20481 ${ }^{1}$ & 42920 & 162946 & 22.33 & 1.19 & & & & \\
\hline HA7Ic02-9271 & 42811.9 & 165733 & 22.33 & 1.23 & 4.03 & -1 & 11 & \\
\hline $\mathrm{HC} 13 \mathrm{c} 09-1062 \mathrm{l}^{1}$ & 42730 & 13517 & 22.36 & 1.22 & & & & \\
\hline HB4Ic08-7561 & 4214.7 & 162245 & 22.36 & 1.26 & 4.73 & 5 & 3 & \\
\hline $\mathrm{HC} 15 \mathrm{c} 10-1701^{1}$ & 43331 & 14945 & 22.41 & 1.19 & & & & \\
\hline HA13c08-15431 & 42911.8 & 145946 & 22.49 & 1.26 & 3.45 & -12 & 6 & \\
\hline HB7Ic04-5581 & 42214.2 & 153118 & 22.51 & 1.27 & 5.86 & -15 & 14 & \\
\hline HB7Ic08-3241 ${ }^{1}$ & 42133 & 15948 & 22.54 & 1.23 & & & & \\
\hline HC06c01-5911 ${ }^{1}$ & 41847 & 183118 & 22.55 & 1.21 & & & & \\
\hline HB7Ic11-7701 & 42240.2 & 151250 & 22.55 & 1.49 & 5.61 & 16 & -46 & \\
\hline HA1Ic09-7351 & 42720.4 & 15480 & 22.56 & 1.27 & 4.64 & 23 & -12 & \\
\hline HC17c02-11961 & 43546.8 & 153941 & 22.58 & 1.25 & 4.88 & -10 & 6 & \\
\hline HB9Ic06-5301 ${ }^{1}$ & 42259 & 141948 & 22.61 & 1.21 & & & & \\
\hline HB9Ic09-4901 ${ }^{1}$ & 42449 & 141832 & 22.61 & 1.22 & & & & \\
\hline HA5Ic01-3851 ${ }^{1}$ & 42934 & 16726 & 22.67 & 1.22 & & & & \\
\hline HB12c11-861 & 4316.1 & 142046 & 22.68 & 1.4 & 4.39 & -56 & 12 & \\
\hline HB18c03-25631 ${ }^{1}$ & 43117 & 171830 & 22.7 & 1.25 & & & & \\
\hline HC18c10-169l & 42714.8 & 131538 & 22.77 & 1.26 & 4.23 & 24 & 12 & \\
\hline HА6Ic02-12021 & 42956 & 163131 & 22.78 & 1.22 & & & & \\
\hline HB1Ic08-16891 & 42537.7 & 172013 & 22.78 & 1.25 & 4.59 & 2 & 0 & \\
\hline HA8Ic00-35321 & 42350 & 165013 & 22.8 & 1.22 & & & & \\
\hline HA7Ic11-22421 & 42943.6 & 164626 & 22.8 & 1.24 & 4.35 & -11 & -35 & \\
\hline HA13c08-18661 & 4296.1 & 15035 & 22.86 & 1.32 & 3.39 & & & \\
\hline HC09c02-15201 & 4170 & 17643 & 22.91 & 1.26 & 4.69 & -7 & -2 & \\
\hline HB6Ic09-3221 & 42153.4 & 153227 & 22.92 & 1.25 & 7.55 & 16 & 10 & \\
\hline HC10c00-261 & 41636.5 & 174326 & 22.97 & 1.26 & 5.18 & -8 & 3 & \\
\hline $\mathrm{HC} 02 \mathrm{c} 11-3411$ & 42442.3 & 174152 & 22.99 & 1.31 & 3.71 & -29 & -35 & \\
\hline
\end{tabular}

${ }^{1}$ This candidate has no follow up $K$-band photometry.

$\dagger$ This candidate has a proper motion consistent with membership but is too blue in $I-K$ to be a cluster member.

$\$$ This extremely red object was also observed in the $J$ and $H$ bands, yielding $(J-H, H-K)=(2.24,0.91)$. 